\title{
Chemical abundance analysis of the open clusters Berkeley 32, NGC 752, Hyades, and Praesepe ${ }^{\star, \star \star, \star \star \star}$
}

\author{
R. Carrera ${ }^{1,2,3,4}$ and E. Pancino ${ }^{3}$
}

\author{
${ }^{1}$ Instituto de Astrofísica de Canarias, La Laguna, Tenerife, Spain \\ e-mail: rcarrera@iac.es \\ 2 Departamento de Astrofísica, Universidad de La Laguna, Tenerife, Spain \\ 3 INAF - Osservatorio Astronomico di Bologna, Bologna, Italy \\ ${ }^{4}$ Centro de Investigaciones de Astronomía, Mérida, Venezuela
}

Received 14 June 2011 / Accepted 12 August 2011

\section{ABSTRACT}

\begin{abstract}
Context. Open clusters are ideal test particles for studying the chemical evolution of the Galactic disc. However, the number and accuracy of existing high-resolution abundance determinations, not only of $[\mathrm{Fe} / \mathrm{H}]$, but also of other key elements, remains largely insufficient.

Aims. We attempt to increase the number of Galactic open clusters that have high quality abundance determinations, and to gather all the literature determinations published so far.

Methods. Using high-resolution $(R \sim 30000)$, high-quality ( $S / N \geq 60$ per pixel), we obtained spectra for twelve stars in four open clusters with the fibre spectrograph FOCES, at the 2.2 Calar Alto Telescope in Spain. We employ a classical equivalent-width analysis to obtain accurate abundances of sixteen elements: $\mathrm{Al}, \mathrm{Ba}, \mathrm{Ca}, \mathrm{Co}, \mathrm{Cr}, \mathrm{Fe}, \mathrm{La}, \mathrm{Mg}, \mathrm{Na}, \mathrm{Nd}, \mathrm{Ni}, \mathrm{Sc}, \mathrm{Si}, \mathrm{Ti}, \mathrm{V}$, and $\mathrm{Y}$. We derived oxygen abundances by means of spectral synthesis of the $6300 \AA$ forbidden line.

Results. We provide the first determination of abundance ratios other than Fe for NGC 752 giants, and ratios in agreement with the literature for the Hyades, Praesepe, and Be 32. We use a compilation of literature data to study Galactic trends of $[\mathrm{Fe} / \mathrm{H}]$ and $[\alpha / \mathrm{Fe}]$ with Galactocentric radius, age, and height above the Galactic plane. We find no significant trends, but some indication for a flattening of $[\mathrm{Fe} / \mathrm{H}]$ at large $R_{\mathrm{gc}}$, and for younger ages in the inner disc. We also detect a possible decrease in $[\mathrm{Fe} / \mathrm{H}]$ with $|\mathrm{z}|$ in the outer disc, and a weak increase in $[\alpha / \mathrm{Fe}]$ with $R_{\mathrm{gc}}$.
\end{abstract}

Key words. Galaxy: disk - open clusters and associations: individual: NGC 752 - open clusters and associations: individual: Hyades open clusters and associations: individual: Berkeley 32 - open clusters and associations: individual: Preasepe (M 44) -

stars: abundances

\section{Introduction}

Open clusters (OC) are ideal test particles for studying the evolution of metallicity with time, inferring the so-called agemetallicity relation, and with Galactocentric radius, the metallicity gradient, measuring in the Galactic disc. Their properties can be determined with smaller uncertainties than for field stars, since they are coeval group of stars at the same distance that have a homogeneous chemical composition. Unfortunately, of the $\simeq 1700$ known OC (e.g., Dias et al. 2002), only $\simeq 140$ possess some metallicity determination, mostly obtained from photometric indicators, such as Washington or Strömgren photometry (see Twarog et al. 1997; Chen et al. 2003, and references therein) and low-resolution spectroscopy (e.g., Friel \& Janes 1993; Friel et al. 2002).

The most accurate way to determine the chemical abundances is to analyse high-resolution spectroscopy. It allows us

\footnotetext{
* Based on observations collected with the fiber spectrograph FOCES at the $2.2 \mathrm{~m}$ Calar Alto Telescope. Also based on data from 2MASS survey and the WEBDA, VALD, NIST, and GEISA online database.

$\star \star$ Table 12 is available in electronic form at http://www . aanda.org

$\star \star \star$ Full Table 5 is only available at the CDS via anonymous ftp to cdsarc.u-strasbg.fr $(130.79 .128 .5)$ or via

http://cdsarc.u-strasbg.fr/viz-bin/qcat?]/A+A/535/A30
}

to investigate not only metallicity, but also abundance ratios with respect to iron or hydrogen - of other chemical species such as $\alpha$-elements, $s$-process elements, and $r$-process elements, which are synthesised in different environments and on different timescales (e.g., SNe Ia, SNe II, giants, supergiants, etc). In the past few years, a number of research groups have addressed the challenge of increasing the number of $\mathrm{OC}$ with chemical abundances determined from high-resolution spectroscopy (e.g., Sestito et al. 2004; D'Orazi et al. 2006; Sestito et al. 2006; Bragaglia et al. 2008; Pace et al. 2008; D’Orazi et al. 2009; Friel et al. 2010; Pace et al. 2010; Pancino et al. 2010a; Jacobson et al. 2011a). However, the number of OC with chemical abundances determined with this technique is still small (see Sect. 5), and significant uncertainties remain in the determinations of both the metallicity gradient and the age-metallicity relation, which are the fundamental ingredients of chemical evolution models.

In this paper, the second of a series initiated by Pancino et al. (2010a, hereafter Paper I), we present high quality and homogeneous measurements of chemical abundances for red clump stars in four OC: Be 32, NGC 752, Hyades, and Praesepe. The Hyades is the nearest $\mathrm{OC}$ and its four known red giants have been widely studied (Schuler et al. 2009; Mishenina et al. 2007; Fulbright et al. 2007; Schuler et al. 2006; Mishenina et al. 2006; Boyarchuk et al. 2000; Luck \& Challener 1995), hence it provides a very good reference frame to compare our abundances 
Table 1. Observing logs and programme star properties.

\begin{tabular}{|c|c|c|c|c|c|c|c|c|c|c|c|}
\hline Cluster & Star & $\begin{array}{c}\alpha_{2000} \\
\text { (h) }\end{array}$ & $\begin{array}{l}\delta_{2000} \\
(\mathrm{deg})\end{array}$ & $\begin{array}{c}B \\
(\mathrm{mag})\end{array}$ & $\begin{array}{c}V \\
(\mathrm{mag})\end{array}$ & $\begin{array}{c}R \\
(\mathrm{mag})\end{array}$ & $\begin{array}{c}I^{a} \\
(\mathrm{mag})\end{array}$ & $\begin{array}{c}K_{\mathrm{S}} \\
(\mathrm{mag})\end{array}$ & $n_{\text {exp }}$ & $\begin{array}{l}t_{\text {exp }}^{\text {tot }} \\
\text { (s) }\end{array}$ & $S / N^{\text {tot }}$ \\
\hline \multirow[t]{2}{*}{$\mathrm{Be} 32^{b}$} & 0456 & $06: 58: 08.2$ & $+06: 24: 19.6$ & 14.76 & 13.67 & - & 12.53 & 11.03 & 7 & 18900 & 60 \\
\hline & 1948 & $06: 58: 04.2$ & $+06: 27: 17.1$ & 14.50 & 13.37 & - & 12.20 & 10.68 & 6 & 16200 & 70 \\
\hline \multirow{4}{*}{ NGC $752^{c}$} & 001 & 01:55:12.6 & $+37: 50: 14.6$ & 10.47 & 9.51 & - & - & 7.23 & 4 & 2400 & 160 \\
\hline & 208 & 01:57:37.6 & $+37: 39: 38.1$ & 10.04 & 8.97 & - & - & 6.41 & 4 & 2400 & 180 \\
\hline & 213 & $01: 57: 38.9$ & $+37: 46: 12.5$ & 10.08 & 9.07 & - & - & 6.68 & 3 & 1800 & 80 \\
\hline & 311 & 01:58:52.9 & $+37: 48: 57.3$ & 10.11 & 9.07 & - & - & 6.64 & 4 & 2400 & 100 \\
\hline Hyades $^{d}$ & $028(\gamma$ tau $)$ & 04:19:47.6 & $+15: 37: 39.5$ & 4.64 & 3.65 & 2.92 & 2.45 & 1.52 & 2 & 120 & 560 \\
\hline \multirow{2}{*}{ (Mel 25) } & $041(\delta$ tau $)$ & $04: 22: 56.1$ & $+17: 32: 33.0$ & 4.75 & 3.76 & 3.03 & 2.56 & 1.64 & 3 & 180 & 450 \\
\hline & $070(\epsilon$ tau $)$ & $04: 28: 37.0$ & $+19: 10: 49.5$ & 4.55 & 3.54 & 2.81 & 2.31 & 1.42 & 3 & 180 & 270 \\
\hline Praesepe $e^{e}$ & 212 & 08:39:50.7 & $+19: 32: 27.0$ & 7.53 & 6.58 & 5.87 & 5.38 & 4.39 & 4 & 240 & 165 \\
\hline (NGC 2632) & 253 & 08:40:06.4 & $+20: 00: 28.1$ & 7.35 & 6.38 & 5.67 & 5.20 & 4.20 & 4 & 240 & 215 \\
\hline (M 44) & 283 & 08:40:22.1 & $+19: 40: 11.9$ & 7.42 & 6.41 & 5.68 & 5.21 & 4.18 & 2 & 120 & 150 \\
\hline
\end{tabular}

Notes. ${ }^{(a)}$ All $I$ magnitudes are in the Johnson system $\left(I_{\mathrm{J}}\right)$ with the exception of those of the Be 32 stars which are in the Cousins system $\left(I_{\mathrm{C}}\right)$. ${ }^{(b)}$ Star names from Richtler \& Sagar (2001); Coordinates, $B, V \& I_{\mathrm{C}}$ magnitudes from D'Orazi et al. (2006); $K_{\mathrm{S}}$ magnitudes from $2 \mathrm{MASS}$. ${ }^{(c)}$ Star names from Heinemann (1926); Coordinates from Høg et al. (2000); $B$ and $V$ magnitudes from Jennens \& Helfer (1975); $K_{\mathrm{S}}$ magnitudes from 2MASS. ${ }^{(d)}$ Star names from van Bueren (1952); Coordinates from Perryman et al. (1997); $B, V, R$ and $I_{\mathrm{J}}$ magnitudes from Johnson et al. (1966); $K_{\mathrm{S}}$ magnitudes from 2MASS. ${ }^{(e)}$ Star names from Klein Wassink (1927); Coordinates from Perryman et al. (1997); $B, V$ and $R$ magnitudes from Coleman (1982); $I_{\mathrm{J}}$ magnitudes from Mendoza (1967); Johnson et al. (1966), $K_{\mathrm{S}}$ magnitudes from 2MASS.

with the literature. Both NGC 752 and Praesepe have been wellstudied, but all information about their chemical composition is based mainly on their main-sequence stars (e.g., Pace et al. 2008; An et al. 2007; Sestito et al. 2004; Burkhart \& Coupry 1998; Hobbs \& Thorburn 1992). To our knowledge, there have been no recent measurements of the chemical abundance of their giants from high-resolution spectroscopy. Finally, Be 32 has been the subject of some studies (e.g., Richtler \& Sagar 2001; Friel et al. 2010; Bragaglia et al. 2008; D’Orazi et al. 2006). The properties and previous studies of each cluster is described in more depth in Sect. 4.

This paper is structured as follows: observations and data reduction are described in Sect. 2; equivalent-width measurements are presented in Sect. 3, together with the abundance analysis and its uncertainties; results are compared with the literature in Sects. 4-6; and finally our main conclusions are summarised in Sect. 7.

\section{Observational material}

A total of twelve stars spread in the four OC were observed. They were selected from the WEBDA ${ }^{1}$ database (Mermilliod 1995), and the $2 \mathrm{MASS}^{2}$ survey (Cutri et al. 2003; Skrutskie et al. 2006). Table 1 summarizes the identifications, coordinates, and magnitudes of each target star. Their position in the color-magnitude diagram taken from D'Orazi et al. (2006), Johnson (1953), Johnson \& Knuckles (1955), and Johnson (1952) for Berkeley 32, NGC 752, Hyades, and Preasepe, respectively, are shown in Fig. 1.

Observations were carried out with the fibre echelle spectrograph FOCES (Pfeiffer et al. 1998) attached at the $2.2 \mathrm{~m}$ Calar Alto Telescope (Almeria, Spain) between the 1 and 3 of January 2005. The chosen set-up provides a spectral resolution

\footnotetext{
${ }^{1}$ http://www univie.ac.at/webda

${ }^{2}$ http://www.ipac.caltech.edu/2mass. 2MASS (Two Micron All Sky Survey) is a joint project of the University of Massachusetts and the Infrared Processing and Analysis Center/California Institute of Technology, funded by the National Aeronautics and Space Administration and the National Science Foundation.
}

$(R=\lambda / \delta \lambda)$ of about 30000 . In summary, all stars were observed in 2-7 exposures lasting 10-30 min each, depending on their magnitudes, until a global signal-to-noise ratio $(S / N)$ of at least 60 per pixel was reached around $6000 \AA$. Exposures with $S / N<20$ were neglected because they were too noisy. Finally, sky exposures as long as our longest exposures (30 min) were taken, but the levels were sufficiently low for us to avoid sky subtraction (as in Paper I). The number of useful exposures, the total integration time, and the global $S / N$ for each star are listed in the last three columns of Table 1.

\subsection{Data reduction}

Various steps of data reduction were performed exactly as in Paper I. Briefly, the frames were de-trended with the IRAF ${ }^{3}$ tasks ccdproc and apflatten. The spectra were then extracted, wavelength-calibrated, normalized, and the echelle orders were merged using tasks in the IRAF echelle package. Finally, the noisy ends of each combined spectrum were cut, allowing for an effective wavelength coverage from 5000 to $9000 \AA$.

Before combining all exposures of each star, we removed sky absorption features (telluric bands of $\mathrm{O}_{2}$ and $\mathrm{H}_{2} \mathrm{O}$ ) with the help of the IRAF task telluric. The same two hot, rapidly rotating stars, HR 3982 and HR 8762, of Paper I were used. The strong $\mathrm{O}_{2}$ band around $7600 \AA$ had been saturated and therefore could not be properly removed. This spectral region was not used for the abundance analysis, in addition to the small gaps between echelle orders that appeared after $\lambda \simeq 8400 \AA$.

\subsection{Radial velocities}

We used DAOSPEC (Stetson \& Pancino 2008) to measure the observed radial velocities for each individual exposure with $S / N \geq 20$, using $\simeq 300$ absorption lines of different elements, with typical uncertainties of about $0.1 \mathrm{~km} \mathrm{~s}^{-1}$ (see Paper I for

\footnotetext{
3 Image Reduction and Analysis Facility, IRAF is distributed by the National Optical Astronomy Observatories, which are operated by the Association of Universities for Research in Astronomy, Inc., under cooperative agreement with the National Science Foundation.
} 
R. Carrera and E. Pancino: Abundances of four open clusters

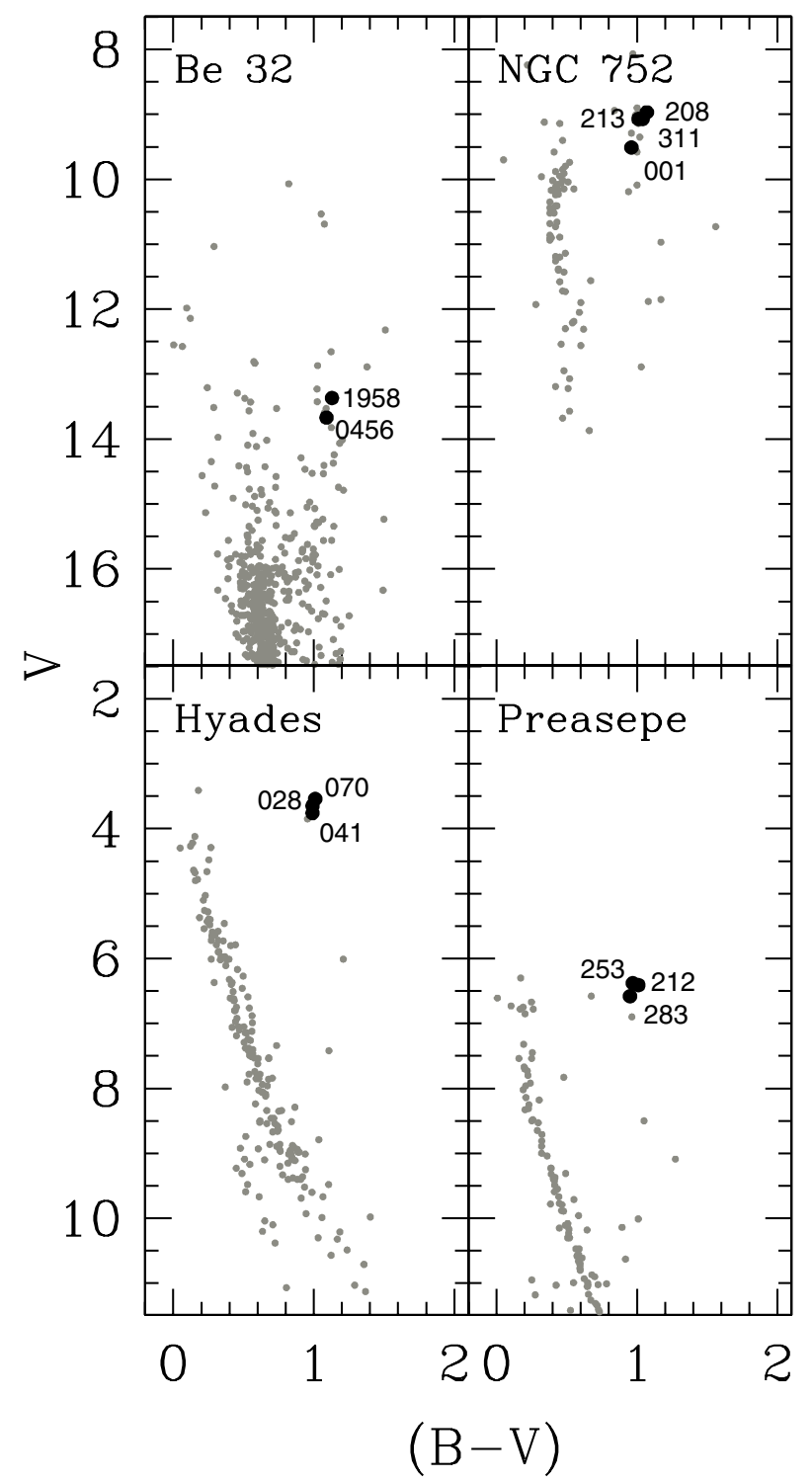

Fig. 1. Location of target stars (large black dots with star ID labels) in the color-magnitude diagrams of their respective parent clusters (small grey dots).

details). We used the same linelist as the one used for abundance determinations (see Sect. 3 for details). Heliocentric corrections were obtained with the IRAF task rvcorrect, with a negligible uncertainty of smaller than $0.005 \mathrm{~km} \mathrm{~s}^{-1}$. We also used DAOSPEC to determine the absolute zero-point of the radial velocity determinations, using a list of telluric absorption lines as the input linelist, obtained from the GEISA ${ }^{4}$ database (Jacquinet-Husson et al. 1999, 2005). The resulting zero-point corrections, based on $\simeq 250$ telluric lines, are generally no larger than $\pm 1 \mathrm{~km} \mathrm{~s}^{-1}$, with a typical error of about $\simeq 0.5 \mathrm{~km} \mathrm{~s}^{-1}$.

The final values, computed as the weighted mean of heliocentric velocities resulting from each exposure of the same star, are listed in Table 2. Our determinations are generally in close agreement with literature values to within $3 \sigma$, except for star 208 in NGC 752, which has a slightly smaller radial velocity than other objects in this cluster. The fact that this star was recognised

\footnotetext{
${ }^{4}$ http://ara.lmd.polytechnique.fr/htdocs-public/ products/GEISA/HTML-GEISA/
}

Table 2. Heliocentric radial velocity measurements and $1 \sigma$ errors $\left(V_{r} \pm\right.$ $\left.\delta V_{r}\right)_{\text {here }}$ for each programme star.

\begin{tabular}{|c|c|c|c|}
\hline Cluster & Star & $\begin{array}{l}\left(V_{r} \pm \delta V_{r}\right)_{\text {here }} \\
\quad\left(\mathrm{km} \mathrm{s}^{-1}\right)\end{array}$ & $\begin{array}{c}\left(V_{r} \pm \sigma V_{r}\right)_{\text {lit }} \\
\left(\mathrm{km} \mathrm{s}^{-1}\right)\end{array}$ \\
\hline \multirow[t]{2}{*}{$\mathrm{Be} 32^{a}$} & 0456 & $105.59 \pm 0.54$ & $110.0 \pm 1.2$ \\
\hline & 1948 & $104.78 \pm 0.35$ & $105.5 \pm 4.9$ \\
\hline \multirow[t]{4}{*}{ NGC $752^{b}$} & 001 & $5.49 \pm 0.44$ & $4.79 \pm 0.15$ \\
\hline & $208^{c}$ & $1.10 \pm 0.23$ & $4.86 \pm 0.06$ \\
\hline & 213 & $5.11 \pm 0.42$ & $5.50 \pm 0.10$ \\
\hline & 311 & $6.00 \pm 0.30$ & $5.28 \pm 0.08$ \\
\hline \multirow{3}{*}{ Hyades $^{d}$} & 028 & $38.15 \pm 0.43$ & $39.28 \pm 0.12$ \\
\hline & 041 & $38.56 \pm 0.36$ & $39.65 \pm 0.08$ \\
\hline & 070 & $38.26 \pm 0.35$ & $39.37 \pm 0.07$ \\
\hline \multirow{3}{*}{ Praesepe $^{e}$ (NGC 2632) } & 212 & $35.96 \pm 0.36$ & $34.81 \pm 0.21$ \\
\hline & 253 & $34.39 \pm 0.27$ & $33.67 \pm 0.22$ \\
\hline & 283 & $34.67 \pm 0.39$ & $34.35 \pm 0.20$ \\
\hline
\end{tabular}

Notes. Literature measurements are also reported with their uncertainties $\left(V_{r} \pm \delta V_{r}\right)_{\text {lit }}$.

References. ${ }^{(a)}$ D’Orazi et al. (2006). ${ }^{(b)}$ Mermilliod et al. (1998). ${ }^{(c)}$ Spectroscopic binary according to Pourbaix et al. (2004). ${ }^{(d)}$ Griffin et al. (1988). ${ }^{(e)}$ Famaey et al. (2005).

as a spectroscopic binary (see Pourbaix et al. 2004; Mermilliod et al. 2007) explains the disagreement. According to its radial velocity curve (Mermilliod et al. 2007), we observed this binary near minimum, which implies that we observed only one of the components of the system. For this reason, and because derived abundances are in good agreement with those of other stars in the same cluster, we retained this object in our final sample. In summary, we considered all the observed targets as likely members of their respective clusters.

\subsection{Photometric parameters}

First guesses of the atmospheric parameters effective temperature $\left(T_{\text {eff }}\right)$, logarithmic gravity $(\log g)$, and microturbulent velocity $\left(v_{\mathrm{t}}\right)$, for our target stars were derived from a photometric data listed in Table 1, as described in Paper I. In brief, $T_{\text {eff }}$ were obtained using the Alonso et al. (1999) and Montegriffo et al. (1998) colour-temperature relations, both theoretical and empirical, and the dereddened colours $(B-V)_{0},\left(V-I_{\mathrm{J}}\right)_{0},(V-R)_{0}$, and $\left(V-K_{\mathrm{S}}\right)_{0}$. We assumed the $E(B-V)$ values listed in Table 3 and the reddening laws of Cardelli et al. (1989). In the case of Be 32 , we have $I_{\mathrm{C}}$ magnitudes instead of $I_{\mathrm{J}}$ ones, so we dereddened $\left(V-I_{\mathrm{C}}\right)$ with the law of Dean et al. (1978), and converted it into $\left(V-I_{\mathrm{J}}\right)_{0}$ with the transformations by Bessell (1979). The $1 \sigma$ errors in each $T_{\text {eff }}$ estimate were computed using the magnitude and reddening uncertainties together with the standard deviation in the colour-temperature relationships used. The photometric $T_{\text {eff }}$ estimates, listed in Table 4, are the weighted mean of the different values obtained from each considered colour and colour-temperature relations.

Photometric gravity estimates were derived from the above $T_{\text {eff }}$ and the bolometric corrections, $\mathrm{BC}_{V}$, derived using the Alonso et al. (1999) prescriptions and the fundamental relationships

$\log \frac{g}{g_{\odot}}=\log \frac{M}{M_{\odot}}+2 \log \frac{R_{\odot}}{R}$
$0.4\left(M_{\mathrm{bol}}-M_{\mathrm{bol}, \odot}\right)=-4 \log \frac{T_{\mathrm{eff}}}{T_{\mathrm{eff}, \odot}}+2 \log \frac{R_{\odot}}{R}$,

where red clump masses, listed in the last column of Table 4, were extrapolated from Table 1 of Girardi \& Salaris (2001). We 
Table 3. Adopted cluster parameters.

\begin{tabular}{lccc}
\hline \hline Cluster & $\begin{array}{c}E(B-V) \\
(\mathrm{mag})\end{array}$ & $\begin{array}{c}(m-M)_{0} \\
(\mathrm{mag})\end{array}$ & $\begin{array}{c}\text { Age } \\
(\mathrm{Gyr})\end{array}$ \\
\hline Be 32 & $0.15 \pm 0.05$ & $12.62 \pm 0.18$ & $4.8 \pm 1.5$ \\
${\text { NGC } 752^{b}}^{a}$ & $0.038 \pm 0.002$ & $8.04 \pm 0.23$ & $1.59 \pm 0.45$ \\
Hyades & $\leq 0.001^{c}$ & $3.34 \pm 0.01^{d}$ & $0.70 \pm 0.07^{e}$ \\
Praesepe & $0.027 \pm 0.004^{c}$ & $6.22 \pm 0.02^{f}$ & $0.65^{g} \pm 0.25$ \\
\hline
\end{tabular}

Notes. When more than one determination exists, the average is shown with $1 \sigma$ errors.

References. ${ }^{(a)}$ Averages of measurements by Kaluzny \& Mazur (1991); Carraro \& Chiosi (1994); Dutra \& Bica (2000); Richtler \& Sagar (2001); Tadross (2001); Lata et al. (2002); Salaris et al. (2004); D'Orazi et al. (2006); and Tosi et al. (2007). ${ }^{(b)}$ Averages of measurements by Johnson (1953); Roman (1955); Johnson (1961); Rohlfs \& Vanysek (1962); Arp (1962); Eggen (1963); Crawford \& Barnes (1970); Patenaude (1978); Hardy (1979); Nicolet (1981); Twarog (1983); Barry et al. (1987); Nissen (1988); Mazzei \& Pigatto (1988); Eggen (1989); Francic (1989); Boesgaard (1991); Dzervitis \& Paupers (1993); Carraro et al. (1993); Meynet et al. (1993); Daniel et al. (1994); Piatti et al. (1995); Dinescu et al. (1995); Milone et al. (1995); Claria et al. (1996); Dutra \& Bica (2000); Loktin \& Beshenov (2001); Blake (2002); Blake \& Rucinski (2004); Salaris et al. (2004); Bartašiūtė et al. (2007); Taylor (2007); and Giardino et al. (2008). (c) Derived by Taylor (2006) from a review of published values. ${ }^{(d)}$ Averages of measurements obtained from the Hipparcos parallaxes by Pinsonneault et al. (1998); Perryman et al. (1998); Narayanan \& Gould (1999); Loktin \& Beshenov (2001); and Percival et al. (2003). ${ }^{(e)}$ Averages of measurements by Eggen (1998); Loktin \& Beshenov (2001); Salaris et al. (2004); Jameson et al. (2008); and Bouvier et al. (2008). (f) Averages of measurements obtained from the Hipparcos parallaxes by Pinsonneault et al. (1998); Perryman et al. (1998); van Leeuwen (1999); Loktin (2000); Loktin \& Beshenov (2001); and Percival et al. (2003). ${ }^{(g)}$ Averages of measurements obtained from the Hipparcos parallaxes by van den Heuvel (1969); Maeder (1971); Mathieu \& Mazeh (1988); Mazzei \& Pigatto (1988); Boesgaard (1989); Tsvetkov (1993); Piatti et al. (1995); Claria et al. (1996); Hernandez et al. (1998); Loktin \& Beshenov (2001); Salaris et al. (2004); Kraus \& Hillenbrand (2007); and Gáspár et al. (2009).

assumed that $\log g_{\odot}=4.437, T_{\mathrm{eff}, \odot}=5770 \mathrm{~K}$ and $M_{\mathrm{bol}, \odot}=4.75$, in conformity with the IAU recommendations (Andersen 1999). As above, we averaged all our estimates to obtain $\log g^{\text {(phot) }}$, listed in Col. 5 of Table 4.

As discussed in Paper I, the photometric estimate of the microturbulent velocity, $v_{\mathrm{t}}$, was obtained using the prescriptions both of Ramírez \& Cohen (2003), $v_{\mathrm{t}}=4.08-5.01 \times 10^{-4} T_{\text {eff }}$, and of Carretta et al. (2004), $v_{\mathrm{t}}=1.5-0.13 \log g$. The latter velocity, which takes into account the effect described by Magain $(1984)^{5}$, is on average lower by $\Delta v_{\mathrm{t}}=0.50 \pm 0.03 \mathrm{~km} \mathrm{~s}^{-1}$ than the Ramírez \& Cohen (2003) estimate. Therefore, we chose not to average the two estimates, but to use them as an indication of the $v_{\mathrm{t}}$ range to explore in our abundance analysis (see Sect. 3.2).

\section{Equivalent widths and abundance analysis}

We used the same linelist as that described in Paper I. In brief, all lines and their atomic data were extracted from the VALD ${ }^{6}$ database (Kupka et al. 1999), with a few exceptions (see Paper I for details). Briefly, for some highly discrepant $\mathrm{Mg}$ lines, we used the NIST $\log g f$ values; we used the Johansson et al. (2003)

\footnotetext{
5 However, see the discussion by Mucciarelli (2011) about the pros and cons of the Magain (1984) correction, which depends heavily on data quality and line selection effects.

${ }^{6}$ http://www. astro.uu.se/ vald/
}

$\log g f$ for the Ni line that contaminates the [O I] line at $6300 \AA$, and provides oxygen abundances more in line with the other $\alpha$-elements; we used the Nd $\log g f$ values by Den Hartog et al. (2003), which minimize the spread in the Nd abundance. Finally, we tried both the VALD and the NIST values for $\mathrm{Ca}$, finding an average difference of 0.17 dex (see Paper I). There is no special reason for choosing NIST over VALD (or vice-versa), so we kept the VALD values to help maintain some homogeneity, but we note that the $\mathrm{Ca} \log g f$ values carry a large uncertainty of the order of 0.2 dex.

\subsection{Equivalent widths with DAOSPEC}

The task DAOSPEC (Stetson \& Pancino 2008) was used to automatically find and measure equivalent widths $(E W)$, by performing a Gaussian fitting of the identified lines. DAOSPEC provides a formal error in the Gaussian fit, $\delta E W$, and a quality parameter, $Q$ (see Stetson \& Pancino 2008; and Paper I, for more details). The relative error $\delta E W / E W$ and the quality parameter $Q$ can be used to distinguish good and bad lines, and they were indeed used to select the highest quality lines for the abundance analysis, as described in detail in Paper I. The measured $E W$ for our program stars are shown in the electronic version of Table 5 along with the $\delta E W$ and $Q$ parameter estimated by DAOSPEC.

Four of our target stars have published $E W$ measurements from high-resolution spectra. These consist of three stars (namely, 028, 041, and 070) observed in the Hyades by Boyarchuk et al. (2000) with $R \sim 45000$, and star 0456 in Be 32 studied by Bragaglia et al. (2008) with $R \sim 40000$. We have a total of 100, 92, and 51 lines in common for stars 028,041 , and 070 in the Hyades, respectively, and 51 lines for star 0456 in Be 32. Figure 2 compares the comparison between the $E W$ determined with DAOSPEC with the values published by Bragaglia et al. (2008) and Boyarchuk et al. (2000). The differences (see Fig. 2) are negligible within the uncertainties; we find a small offset of $5.6 \mathrm{~m} \AA$ in the case of star 041 in the Hyades, which is however still within $1 \sigma$. We can therefore consider our measurements in good agreement with similar studies.

\subsection{Abundance analysis}

Abundance calculations and spectral synthesis (for oxygen) were performed using the latest version of the abundance calculation code originally described by Spite (1967). We used the MARCS model atmospheres developed by Edvardsson et al. (1993). We also used of ABOMAN, a tool developed by E. Rossetti at the INAF, Bologna Observatory, Italy, which allows the semi-automatic processing of data for several objects, using the aforementioned abundance calculation code. The tool ABOMAN performs all the steps needed to choose the best-fit model automatically (see below) and compute abundance ratios for all elements, and provides all the graphical tools required to analyse the results.

The detailed procedure followed to derive the chemical abundances is described in depth in Paper I. In brief, we calculated Fe I and Fe II abundances for a set of models with parameters extending $\pm 3 \sigma$ around the photometric estimates of Table 4 . We chose the model that satisfied simultaneously the following conditions: (i) the abundance of Fe I lines should not vary with excitation potential $\chi_{\text {ex }}$; (ii) the abundance of Fe I lines should not vary significantly with $E W$, i.e., strong and weak 
Table 4. Stellar atmosphere parameters for the programme stars (see text).

\begin{tabular}{lcccccccc}
\hline \hline Cluster & Star & $\begin{array}{c}T_{\text {eff }}^{\text {(phot) }} \\
(\mathrm{K})\end{array}$ & $\begin{array}{c}T_{\text {eff }}^{(\text {(spec) }} \\
(\mathrm{K})\end{array}$ & $\begin{array}{c}\log g^{\text {(phot) }} \\
(\mathrm{cgs})\end{array}$ & $\begin{array}{c}\log g^{(\text {spec })} \\
(\mathrm{cgs})\end{array}$ & $\begin{array}{c}v_{\mathrm{t}}^{\text {(phot) }} \\
\left(\mathrm{km} \mathrm{s}^{-1}\right)\end{array}$ & $\begin{array}{c}v_{\mathrm{t}}^{\text {(spec) }} \\
\left(\mathrm{km} \mathrm{s}^{-1}\right)\end{array}$ & $\begin{array}{c}M_{\text {clump }} \\
\left(M_{\odot}\right)\end{array}$ \\
\hline Be 32 & 0456 & $4759 \pm 92$ & 4650 & $2.61 \pm 0.14$ & 2.1 & $1.70 \pm 0.30 / 1.16 \pm 0.10$ & 1.4 & $1.2 \pm 0.1$ \\
& 1948 & $4706 \pm 99$ & 4700 & $2.47 \pm 0.14$ & 2.3 & $1.72 \pm 0.30 / 1.18 \pm 0.10$ & 1.5 & $1.2 \pm 0.1$ \\
NGC 752 & 001 & $4949 \pm 80$ & 5050 & $3.02 \pm 0.14$ & 3.1 & $1.60 \pm 0.30 / 1.11 \pm 0.10$ & 1.3 & $1.9 \pm 0.2$ \\
& 208 & $4698 \pm 110$ & 4600 & $2.73 \pm 0.14$ & 2.9 & $1.73 \pm 0.31 / 1.15 \pm 0.10$ & 1.2 & $1.9 \pm 0.2$ \\
& 213 & $4841 \pm 86$ & 4900 & $2.81 \pm 0.14$ & 3.0 & $1.65 \pm 0.30 / 1.13 \pm 0.10$ & 1.4 & $1.9 \pm 0.2$ \\
& 311 & $4793 \pm 74$ & 4800 & $2.80 \pm 0.14$ & 3.2 & $1.68 \pm 0.30 / 1.14 \pm 0.10$ & 1.2 & $1.9 \pm 0.2$ \\
Hyades & 028 & $4865 \pm 73$ & 4750 & $2.67 \pm 0.04$ & 2.7 & $1.64 \pm 0.30 / 1.15 \pm 0.15$ & 1.4 & $2.5 \pm 0.1$ \\
& 041 & $4871 \pm 79$ & 4800 & $2.71 \pm 0.05$ & 2.8 & $1.64 \pm 0.30 / 1.15 \pm 0.15$ & 1.4 & $2.5 \pm 0.1$ \\
& 070 & $4858 \pm 95$ & 4800 & $2.62 \pm 0.05$ & 2.8 & $1.65 \pm 0.30 / 1.16 \pm 0.15$ & 1.6 & $2.5 \pm 0.1$ \\
Praesepe & 212 & $4901 \pm 35$ & 4900 & $2.70 \pm 0.07$ & 2.8 & $1.62 \pm 0.30 / 1.15 \pm 0.14$ & 1.5 & $2.6 \pm 0.3$ \\
& 253 & $4869 \pm 23$ & 4900 & $2.60 \pm 0.07$ & 2.8 & $1.64 \pm 0.30 / 1.16 \pm 0.14$ & 1.6 & $2.6 \pm 0.3$ \\
& 283 & $4841 \pm 29$ & 4800 & $2.61 \pm 0.07$ & 2.9 & $1.65 \pm 0.30 / 1.16 \pm 0.14$ & 1.4 & $2.6 \pm 0.3$ \\
\hline
\end{tabular}

Table 5. Equivalent widths and atomic data of the programme stars.

\begin{tabular}{|c|c|c|c|c|c|c|c|c|c|c|c|c|c|}
\hline \multirow[b]{2}{*}{$\begin{array}{l}\lambda \\
(\mathrm{A})\end{array}$} & \multirow[b]{2}{*}{ Elem } & \multirow[b]{2}{*}{$\begin{array}{l}\chi_{\mathrm{ex}} \\
(\mathrm{eV})\end{array}$} & \multirow[b]{2}{*}{$\begin{array}{r}\log g f \\
(\operatorname{dex})\end{array}$} & \multicolumn{3}{|c|}{ Be 32-Star 0456} & \multicolumn{3}{|c|}{ Be 32-Star 1948} & \multirow{2}{*}{$\begin{array}{l}\ldots \\
\ldots \\
\ldots\end{array}$} & \multicolumn{3}{|c|}{ Praesepe-Star 283} \\
\hline & & & & $\begin{array}{c}E W \\
(\mathrm{~m} \AA)\end{array}$ & $\begin{array}{l}\delta E W \\
(\mathrm{~m} \AA)\end{array}$ & $Q$ & $\begin{array}{c}E W \\
(\mathrm{~m} \AA)\end{array}$ & $\begin{array}{l}\delta E W \\
(\mathrm{~m} \AA)\end{array}$ & $Q$ & & $\begin{array}{c}E W \\
(\mathrm{~m} \AA)\end{array}$ & $\begin{array}{l}\delta E W \\
(\mathrm{~m} \AA)\end{array}$ & $Q$ \\
\hline 6696.79 & AL1 & 4.02 & -1.42 & 16.5 & 10 & 0.361 & 25.3 & 3.9 & 0.481 & & 30.0 & 5.0 & 0.585 \\
\hline & & & & & & & & & & & 9.2 & & .321 \\
\hline & & & & & & & & & & & 52.1 & 2.1 & 0.173 \\
\hline 736 & $\mathrm{AL}$ & 4.0 & & 48 & 4. & & & 2.9 & 0.737 & & 72.0 & 6.9 & 0.797 \\
\hline 7835.31 & AL1 & 4.02 & -0.65 & 53.7 & 3.7 & 0.393 & 48.4 & 3.9 & 0.611 & $\ldots$ & 95.8 & 9.8 & 1.399 \\
\hline 7836.13 & AL1 & 4.0 & -0.49 & 66.0 & 7.8 & 1.279 & 68.4 & 5.1 & 0.783 & & 94.0 & 6.0 & 0.665 \\
\hline 8772.86 & AL1 & 4.02 & -0.32 & & $\cdots$ & & & $\ldots$ & & $\ldots$ & 102.7 & 8.7 & 0.748 \\
\hline 8773.90 & AL1 & 4.02 & -0.16 & 115.0 & 8.6 & 1.151 & 111.9 & 8.3 & 1.111 & $\ldots$ & & & \\
\hline 5853.67 & BA2 & 0.60 & -1.00 & 119.7 & 3.9 & 1.098 & 102.0 & 2.6 & 0.343 & $\ldots$ & 122.1 & 3.4 & 0.676 \\
\hline
\end{tabular}

Notes. The complete version of the table is available at the CDS. Here we show a few lines to illustrate its contents.

lines should infer the same abundance ${ }^{7}$; (iii) the abundance of Fe I lines should not differ significantly from the abundance of Fe II lines; and (iv) the abundance of Fe I lines should not vary significantly with wavelength.

Once the best-fit model has been found, abundance ratios of all the measured elements were determined, as shown in Table 6 , as the average of abundances given by single lines. The internal (random) errors were then computed as $\left.\sigma / \sqrt{(} n_{\text {lines }}\right)$. Oxygen abundances were determined by means of spectral synthesis of the region around the $[\mathrm{O}$ I] forbidden line at $6300 \AA$. In this case, the internal uncertainty was estimated using the average abundance difference between the best-fit spectrum and two spectra placed approximately $1 \sigma$ (of the Poissonian noise) above and below it. Average cluster abundances (Tables 7) were computed as weighted averages of abundance ratios of single stars.

Comparison of our results with available literature is discussed in details in Sect. 4.

\subsection{Abundance uncertainties and the Sun}

The internal (random) uncertainty described above includes uncertainties related to the measurement of $E W$ and to the atomic parameters (dominated by $\log g f$ determinations). We must consider other sources of uncertainty (see Paper I for details) such as: the uncertainty owing to the choice of atmospheric parameters; the uncertainty owing to the continuum normalization procedure; the uncertainty in the reference solar abundance values.

\footnotetext{
7 We decided not to use the Magain (1984) effect, because we prefer to have internally consistent abundances from each line, and because of the additional effects described by Mucciarelli (2011).
}

Uncertainties due to the choice of stellar parameters were evaluated with the method proposed by Cayrel et al. (2004). In brief, we altered the predominant atmospheric parameter, i.e., by altering one atmospheric parameter, $T_{\text {eff }}$, within its uncertainty $(\sim 100 \mathrm{~K})$ and re-optimizing the other parameters for the hottest and coolest stars in our sample. We re-calculated abundances with the procedure described in the previous Section. The external uncertainties, listed in the last column of Table 6, are estimated by averaging errors calculated with the higher and lower temperatures for the warmest and coolest stars in our sample (namely, stars 001 and 208 in NGC 752).

Uncertainties due to the continuum normalization procedure might also affect the obtained $E W$ and, therefore, the derived abundances. Their contribution is estimated by averaging the differences between the $E W$ obtained with the "best-fit" continuum and those derived by lowering and raising the continuum level by the continuum placement uncertainty. This is calculated from Eq. (7) of Stetson \& Pancino (2008). The typical uncertainty caused by the continuum placement is $\Delta E W \sim 1 \mathrm{~m} \AA$ and almost independent of the $E W$. This small uncertainty has a negligible impact on the derived abundances in comparison with other sources of uncertainty described above. Therefore, they have not been explicitly included in the error budget.

To validate the whole procedure used here, in Paper I we performed an abundance analysis of the ESO HARPS solar spectrum reflected by Ganymede ${ }^{8}$. We used the same line list, model atmospheres, and abundance calculation code that we used on our OC target stars, and found solar values for all elements,

\footnotetext{
8 http://www.1s.eso.org/lasilla/sciops/3p6/harps/ monitoring/sun.html
} 


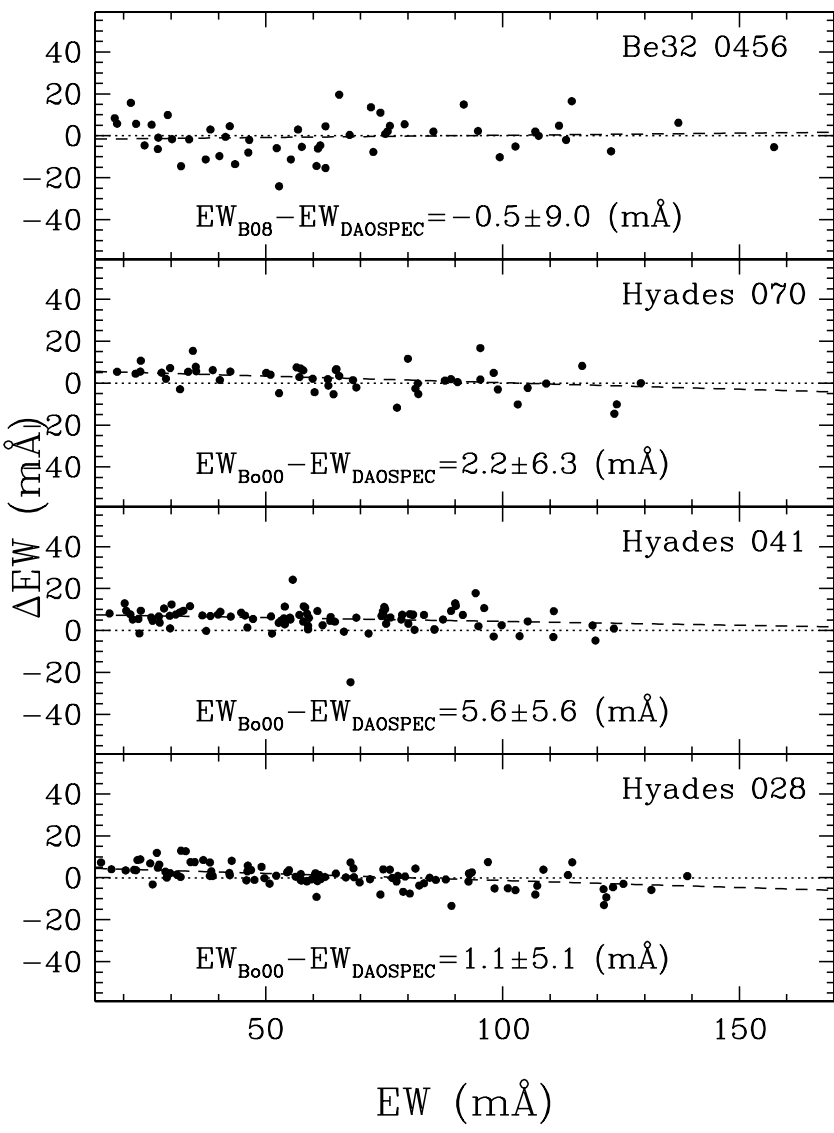

Fig. 2. Comparison of our $E W$ measurements with those by Bragaglia et al. (2008) for star 0456 in Be 32, and by Boyarchuk et al. (2000) for three Hyades giants. Dotted lines mark perfect agreement (zero difference), while dashed lines are linear fits to the data.

with the only marginal exceptions of barium and aluminium (see also Sect. 5). While the details of this analysis can be found in Paper I, we mention here that our reference solar abundances are taken from Grevesse et al. (1996).

\section{Cluster-by-cluster discussion}

\subsection{Berkeley 32}

Berkeley $32\left(\alpha_{2000}=06^{\mathrm{h}} 58^{\mathrm{m}} 07^{\mathrm{s}}\right.$ and $\left.\delta_{2000}=+06^{\circ} 25^{\prime} 43^{\prime \prime}\right)$ is a distant OC $\left(R_{\mathrm{gc}}=11.6 \mathrm{kpc}\right)$ located towards the Galactic anticentre and situated $260 \mathrm{pc}$ above the disc plane. Its distance makes it one of the crucial clusters for a correct determination of the metallicity gradient along the Galactic disc, and therefore one of the key OC to the understanding of disc formation and evolution. The color-magnitude diagram of this cluster (e.g., D'Orazi et al. 2006), contaminated by disc stars, shows a clear main sequence turn-off with a sparsely populated red giant branch. Determinations of its age, mainly using morphological indicators, yield a value of $\simeq 5$ Gyr (e.g., D'Orazi et al. 2006; Salaris et al. 2004; Richtler \& Sagar 2001; Carraro \& Chiosi 1994; Kaluzny \& Mazur 1991).

Given its large distance, it has not been well-studied spectroscopically, but we could compare our results with two recent high-resolution studies of Bragaglia et al. (2008) and Friel et al. (2010). We found a very close agreement of our abundance ratios with those studies (see Table 8). The exceptions are $\mathrm{Ba}$ and $\mathrm{Na}$. It is well-known that $\mathrm{Ba}$ abundances are enhanced by HFS (e.g., D'Orazi et al. 2009) effects that should explain the differences from Bragaglia et al. (2008). The $[\mathrm{Na} / \mathrm{Fe}]$ ratio is lower than the values reported by Bragaglia et al. (2008) and Friel et al. (2010) by -0.25 and -0.32 dex, respectively. The difficulty in measuring Na lines, which suffer from NLTE effects, could easily explain this controversy. Moreover, different model atmospheres, stellar and atomic parameters, etc., between different studies may also play a role (and remove this discrepancy).

\subsection{NGC 752}

NGC $752\left(\alpha_{2000}=01^{\mathrm{h}} 57^{\mathrm{m}} 41^{\mathrm{s}}, \delta_{2000}=+37^{\circ} 47^{\prime} 06^{\prime \prime}\right)$ is an old $(\sim 1.6 \mathrm{Gyr})$ OC located in the solar neighbourhood at a distance of $\simeq 400 \mathrm{pc}$. This cluster has a low central concentration and contains a relatively small number of members. Its color-magnitude diagram (e.g., Johnson 1953) has a still poorly understood morphology. The turn-off area is well-populated by early F-type stars, while the low main-sequence appears to be sparsely populated (Fig. 1). This, together with the age of this cluster, may be an indication of the dynamic escape of low mass stars. Stellar evolution models also predict a well-populated red giant branch, which is not observed. All the known red giants are located in the red clump region (Bartašiūtè et al. 2007), which has a peculiar morphology because it has a faint extension slightly to the blue of its main concentration, which cannot be reproduced by stellar evolution models (Girardi et al. 2000).

Photometry and low/medium resolution spectroscopy studies (see Bartašiūtė et al. 2007, and references therein) determined a slightly subsolar metallicity (i.e. $[\mathrm{Fe} / \mathrm{H}]=-0.16 \pm 0.05$, Friel \& Janes 1993). A similar result was found with high-resolution spectroscopy $(R \simeq 40000, S / N \simeq 80-150)$ in eight F-type stars around the main sequence turn-off $([\mathrm{Fe} / \mathrm{H}]=-0.09 \pm 0.05$, Hobbs \& Thorburn 1992). However, an investigation based on high-resolution spectroscopy $(R \simeq 57000$, $S / N \simeq 30-80$ ) of $18 \mathrm{G}$ giant stars obtained a solar $[\mathrm{Fe} / \mathrm{H}]$ ratio $([\mathrm{Fe} / \mathrm{H}]=+0.01 \pm 0.04$, Sestito et al. 2004) in closer agreement with the value determined here. To our knowledge, we are the first to publish abundance ratios of elements other than $[\mathrm{Fe} / \mathrm{H}]$ for this cluster.

\subsection{Hyades}

The Hyades cluster (Melotte $25 ; \alpha_{2000}=04^{\mathrm{h}} 26^{\mathrm{m}} 54^{\mathrm{s}}$ and $\delta_{2000}=$ $\left.+15^{\circ} 52^{\prime} 00^{\prime \prime}\right)$ is the closest OC to the Sun $(\sim 45 \mathrm{pc})$ located in the constellation of Taurus. Its proximity has motivated an extensive study lasting more than a century (starting with Hertzsprung 1909). The OC is embedded into a moving group with the same name, which suggests that it would be part of a dynamical stream coming from the inner Galaxy or a disrupting cluster (Famaey et al. 2007).

Being one of the most studied clusters, both photometrically and spectroscopically, it is the ideal cluster for abundance analysis comparisons. The color-magnitude diagram of this young OC ( 0.7 Gyr, see Table 3; e.g., Johnson \& Knuckles 1955) contains only four red giant stars that have been confirmed as members from their parallaxes, proper motions, and radial velocities. Most of the existing abundance studies are focused on main sequence stars (see e.g., Paulson et al. 2003; Burkhart \& Coupry 2000; Varenne \& Monier 1999, and references therein). A comparison of the Hyades average abundances determined from some (or all) of the known four red giants are shown in Table 10. The averages of the abundances compiled until 1999 by Varenne \& Monier (1999) are shown in the last column of Table 10 for reference. In general, $[\mathrm{Fe} / \mathrm{H}]$ appears slightly 
R. Carrera and E. Pancino: Abundances of four open clusters

Table 6. Abundance ratios for single cluster stars, with their internal and external (last column) uncertainties.

\begin{tabular}{|c|c|c|c|c|c|c|c|}
\hline \multirow[b]{2}{*}{ Ratio } & \multicolumn{2}{|c|}{ Berkeley 32} & \multicolumn{4}{|c|}{ NGC 752} & \multirow{2}{*}{$\begin{array}{c}\text { External } \\
\text { Uncertainty }\end{array}$} \\
\hline & Star 456 & Star 1948 & Star 001 & Star 208 & Star 213 & Star 311 & \\
\hline$[\mathrm{Fel} / \mathrm{H}]$ & $-0.33 \pm 0.02$ & $-0.27 \pm 0.02$ & $+0.07 \pm 0.01$ & $+0.07 \pm 0.01$ & $+0.04 \pm 0.01$ & $+0.14 \pm 0.01$ & \pm 0.03 \\
\hline$[\mathrm{FeII} / \mathrm{H}]$ & $-0.30 \pm 0.06$ & $-0.29 \pm 0.06$ & $+0.02 \pm 0.03$ & $+0.06 \pm 0.03$ & $+0.05 \pm 0.04$ & $+0.18 \pm 0.12$ & \pm 0.03 \\
\hline$[\alpha / \mathrm{Fe}]$ & $-0.29 \pm 0.21$ & $-0.25 \pm 0.09$ & $+0.07 \pm 0.04$ & $+0.05 \pm 0.12$ & $+0.07 \pm 0.12$ & $+0.14 \pm 0.09$ & \pm 0.07 \\
\hline$[\mathrm{Al} / \mathrm{Fe}]$ & $+0.15 \pm 0.06$ & $+0.08 \pm 0.07$ & $-0.11 \pm 0.04$ & $-0.12 \pm 0.03$ & $-0.06 \pm 0.06$ & $-0.21 \pm 0.06$ & \pm 0.05 \\
\hline$[\mathrm{Ba} / \mathrm{Fe}]$ & $+0.52 \pm 0.05$ & $+0.35 \pm 0.17$ & $+0.55 \pm 0.04$ & $+0.52 \pm 0.04$ & $+0.51 \pm 0.01$ & $+0.57 \pm 0.06$ & \pm 0.04 \\
\hline$[\mathrm{Ca} / \mathrm{Fe}]$ & $-0.06 \pm 0.08$ & $-0.05 \pm 0.04$ & $-0.02 \pm 0.03$ & $-0.12 \pm 0.02$ & $-0.09 \pm 0.03$ & $-0.17 \pm 0.05$ & 0.06 \\
\hline$[\mathrm{Co} / \mathrm{Fe}]$ & $+0.02 \pm 0.05$ & $+0.09 \pm 0.04$ & $-0.03 \pm 0.03$ & $+0.06 \pm 0.04$ & $+0.00 \pm 0.03$ & $+0.05 \pm 0.05$ & 0.04 \\
\hline$[\mathrm{Cr} / \mathrm{Fe}]$ & $-0.25 \pm 0.07$ & $+0.04 \pm 0.07$ & $+0.02 \pm 0.03$ & $+0.00 \pm 0.03$ & $-0.01 \pm 0.03$ & -0.01 & 0.05 \\
\hline$[\mathrm{La} / \mathrm{Fe}]$ & $-0.14 \pm 0.02$ & $-0.04 \pm 0.08$ & $+0.14 \pm 0.06$ & $+0.18 \pm 0.03$ & $+0.18 \pm 0.09$ & $+0.32 \pm 0.13$ & \pm 0.04 \\
\hline$[\mathrm{Mg} / \mathrm{Fe}]$ & $+0.38 \pm 0.12$ & $+0.24 \pm 0.16$ & $+0.13 \pm 0.06$ & $+0.16 \pm 0.05$ & $+0.20 \pm 0.04$ & $+0.06 \pm 0.03$ & \pm 0.09 \\
\hline$[\mathrm{Na} / \mathrm{Fe}]$ & $-0.14 \pm 0.08$ & $-0.08 \pm 0.10$ & $+0.05 \pm 0.01$ & $-0.07 \pm 0.02$ & $-0.03 \pm 0.05$ & $-0.10 \pm 0.05$ & \pm 0.04 \\
\hline$[\mathrm{Nd} / \mathrm{Fe}]$ & $-0.05 \pm 0.13$ & $+0.04 \pm 0.03$ & $+0.29 \pm 0.14$ & $+0.27 \pm 0.23$ & $+0.34 \pm 0.11$ & \pm 0.18 & \pm 0.13 \\
\hline$[\mathrm{Ni} / \mathrm{Fe}]$ & $-0.04 \pm 0.03$ & $-0.01 \pm 0.03$ & $-0.04 \pm 0.02$ & $+0.00 \pm 0.02$ & $-0.02 \pm 0.02$ & $+0.03 \pm 0.03$ & \pm 0.02 \\
\hline$[\mathrm{O} / \mathrm{Fe}]$ & $-0.16 \pm 0.13$ & $+0.15 \pm 0.11$ & $+0.15 \pm 0.06$ & $-0.06 \pm 0.05$ & $+0.02 \pm 0.08$ & $+0.00 \pm 0.06$ & \pm 0.08 \\
\hline$[\mathrm{Sc} / \mathrm{Fe}]$ & $+0.02 \pm 0.05$ & $-0.02 \pm 0.05$ & & \pm 0.06 & $+0.05 \pm 0.06$ & & \pm 0.05 \\
\hline$[\mathrm{Si} / \mathrm{Fe}]$ & $+0.18 \pm 0.04$ & $+0.11 \pm 0.04$ & 0.03 & $+0.04 \pm 0.03$ & $+0.04 \pm 0.03$ & 0.04 & \pm 0.04 \\
\hline$[\mathrm{TiI} / \mathrm{Fe}]$ & $-0.10 \pm 0.05$ & $-0.04 \pm 0.05$ & $+0.00 \pm 0.02$ & $-0.03 \pm 0.02$ & $-0.08 \pm 0.02$ & & \pm 0.03 \\
\hline [TiII/Fe] & $-0.17 \pm 0.05$ & $+0.01 \pm 0.07$ & $+0.03 \pm$ & $+0.08 \pm 0.07$ & $+0.07 \pm 0.06$ & +0 & \\
\hline$[\mathrm{V} / \mathrm{Fe}]$ & $-0.14 \pm 0.10$ & $-0.07 \pm 0.05$ & 02 & $+0.16 \pm 0.05$ & -0.04 & +0 & \pm 0.06 \\
\hline \multirow[t]{2}{*}[\mathrm{Y}/\mathrm{Fe}]{} & $-0.41 \pm$ N.A. & $-0.09 \pm$ N.A. & .06 & \pm 0.10 & $+0.03 \pm 0.07$ & +0 & \pm 0.04 \\
\hline & & Hyades (Mel 25) & \multicolumn{5}{|c|}{ Praesepe (NGC 2632) } \\
\hline Ratio & Star 28 & Star 41 & Star 70 & Star 212 & Star 253 & Sta & \\
\hline$[\mathrm{Fel} / \mathrm{H}]$ & $+0.12 \pm 0.01$ & $+0.10 \pm 0.01$ & $0.11 \pm 0.01$ & $+0.11 \pm 0.01$ & $+0.18 \pm 0.01$ & $+0.21 \pm 0.01$ & .03 \\
\hline$[\mathrm{FeII} / \mathrm{H}]$ & $+0.13 \pm 0.03$ & +0.13 & +0.09 & $+0.10 \pm 0.03$ & +0.1 & +0.23 & .03 \\
\hline$[\alpha / \mathrm{Fe}]$ & $+0.13 \pm 0.15$ & $+0.11 \pm 0.12$ & $+0.09 \pm 0.11$ & $+0.11 \pm 0.15$ & $+0.18 \pm 0.14$ & +0.19 & \pm 0.07 \\
\hline$[\mathrm{Al} / \mathrm{Fe}]$ & $-0.01 \pm 0.05$ & $+0.00 \pm 0.05$ & $+0.02 \pm 0.05$ & $+0.01 \pm 0.04$ & $+0.02 \pm 0.06$ & $-0.04 \pm 0.05$ & \pm 0.05 \\
\hline$[\mathrm{Ba} / \mathrm{Fe}]$ & $+0.37 \pm 0.05$ & $+0.39 \pm 0.05$ & $+0.31 \pm 0.05$ & $+0.30 \pm 0.08$ & $+0.27 \pm 0.06$ & $+0.37 \pm 0.05$ & \pm 0.04 \\
\hline$[\mathrm{Ca} / \mathrm{Fe}]$ & $-0.07 \pm 0.03$ & $-0.06 \pm 0.03$ & $-0.07 \pm 0.02$ & $-0.07 \pm 0.02$ & $-0.08 \pm 0.03$ & $-0.11 \pm 0.03$ & \pm 0.06 \\
\hline$[\mathrm{Co} / \mathrm{Fe}]$ & $+0.00 \pm 0.04$ & $+0.01 \pm 0.03$ & $+0.06 \pm 0.03$ & $+0.05 \pm 0.03$ & $+0.01 \pm 0.03$ & $+0.05 \pm 0.05$ & \pm 0.04 \\
\hline$[\mathrm{Cr} / \mathrm{Fe}]$ & $+0.02 \pm 0.03$ & $+0.03 \pm 0.03$ & $+0.08 \pm 0.04$ & $+0.06 \pm 0.03$ & $+0.04 \pm 0.04$ & $+0.04 \pm 0.04$ & \pm 0.05 \\
\hline$[\mathrm{La} / \mathrm{Fe}]$ & $-0.12 \pm 0.06$ & $-0.08 \pm 0.05$ & $-0.05 \pm 0.05$ & $-0.07 \pm 0.05$ & $-0.04 \pm 0.05$ & $-0.04 \pm 0.04$ & \pm 0.04 \\
\hline$[\mathrm{Mg} / \mathrm{Fe}]$ & $+0.13 \pm 0.05$ & $+0.06 \pm 0.04$ & $+0.21 \pm 0.07$ & $+0.31 \pm 0.06$ & $+0.27 \pm 0.05$ & $+0.22 \pm 0.06$ & \pm 0.09 \\
\hline$[\mathrm{Na} / \mathrm{Fe}]$ & $+0.19 \pm 0.02$ & $+0.18 \pm 0.02$ & $+0.18 \pm 0.02$ & $+0.23 \pm 0.02$ & $+0.30 \pm 0.03$ & $+0.18 \pm 0.05$ & \pm 0.04 \\
\hline$[\mathrm{Nd} / \mathrm{Fe}]$ & $+0.04 \pm 0.29$ & $+0.08 \pm 0.30$ & $+0.08 \pm 0.28$ & $+0.00 \pm 0.21$ & $+0.05 \pm 0.25$ & $+0.10 \pm 0.31$ & \pm 0.13 \\
\hline$[\mathrm{Ni} / \mathrm{Fe}]$ & $+0.02 \pm 0.02$ & $+0.04 \pm 0.02$ & $+0.03 \pm 0.02$ & $+0.01 \pm 0.02$ & $+0.01 \pm 0.02$ & $+0.04 \pm 0.03$ & \pm 0.02 \\
\hline$[\mathrm{O} / \mathrm{Fe}]$ & $-0.35 \pm 0.07$ & $-0.25 \pm 0.05$ & $-0.22 \pm 0.07$ & $-0.11 \pm 0.09$ & $-0.14 \pm 0.07$ & $-0.09 \pm 0.06$ & \pm 0.08 \\
\hline$[\mathrm{Sc} / \mathrm{Fe}]$ & $-0.04 \pm 0.05$ & $+0.00 \pm 0.05$ & $-0.02 \pm 0.06$ & $-0.10 \pm 0.06$ & $+-0.03 \pm 0.05$ & $+0.00 \pm 0.06$ & \pm 0.05 \\
\hline$[\mathrm{Si} / \mathrm{Fe}]$ & $+0.09 \pm 0.03$ & $+0.09 \pm 0.02$ & $+0.10 \pm 0.03$ & $+0.06 \pm 0.03$ & $+0.07 \pm 0.03$ & $+0.04 \pm 0.03$ & \pm 0.04 \\
\hline [TiI/Fe] & $-0.12 \pm 0.02$ & $-0.11 \pm 0.02$ & $-0.06 \pm 0.02$ & $-0.05 \pm 0.03$ & $-0.08 \pm 0.02$ & $-0.09 \pm 0.02$ & \pm 0.03 \\
\hline [TiII/Fe] & $-0.03 \pm 0.06$ & $+0.00 \pm 0.07$ & $-0.02 \pm 0.11$ & $-0.05 \pm 0.10$ & $-0.02 \pm 0.08$ & $+0.05 \pm 0.08$ & \pm 0.03 \\
\hline & $+0.02 \pm 0.04$ & $+0.00 \pm 0.03$ & $+0.09 \pm 0.03$ & $+0.06 \pm 0.04$ & $+0.04 \pm 0.03$ & $+0.10=$ & \pm 0.06 \\
\hline$[\mathrm{Y} / \mathrm{Fe}]$ & $-0.12 \pm 0.05$ & $-0.06 \pm 0.06$ & $-0.07 \pm 0.05$ & $-0.11 \pm 0.10$ & $-0.12 \pm 0.07$ & $-0.11 \pm 0.09$ & \pm 0.04 \\
\hline
\end{tabular}

Table 7. Average cluster abundances, obtained as the weighted average of the single stars abundances in each of them.

\begin{tabular}{lcccc}
\hline \hline Ratio & Be 32 & NGC 752 & Hyades & Praesepe (M 44) \\
\hline$[\mathrm{Fe} / \mathrm{H}]$ & $-0.30 \pm 0.02( \pm 0.03)$ & $+0.08 \pm 0.04( \pm 0.03)$ & $+0.11 \pm 0.01( \pm 0.03)$ & $+0.16 \pm 0.05( \pm 0.03)$ \\
{$[\alpha / \mathrm{Fe}]$} & $-0.04 \pm 0.14( \pm 0.07)$ & $+0.02 \pm 0.06( \pm 0.07)$ & $+0.00 \pm 0.12( \pm 0.07)$ & $+0.00 \pm 0.14( \pm 0.07)$ \\
\hline$[\mathrm{Al} / \mathrm{Fe}]$ & $+0.12 \pm 0.05( \pm 0.05)$ & $-0.12 \pm 0.06( \pm 0.05)$ & $+0.00 \pm 0.02( \pm 0.05)$ & $+0.00 \pm 0.03( \pm 0.05)$ \\
{$[\mathrm{Ba} / \mathrm{Fe}]$} & $+0.51 \pm 0.12( \pm 0.04)$ & $+0.52 \pm 0.03( \pm 0.04)$ & $+0.36 \pm 0.04( \pm 0.04)$ & $+0.33 \pm 0.05( \pm 0.04)$ \\
{$[\mathrm{Ca} / \mathrm{Fe}]$} & $-0.05 \pm 0.01( \pm 0.06)$ & $-0.09 \pm 0.06( \pm 0.06)$ & $-0.07 \pm 0.01( \pm 0.06)$ & $-0.08 \pm 0.02( \pm 0.06)$ \\
{$[\mathrm{Co} / \mathrm{Fe}]$} & $+0.07 \pm 0.05( \pm 0.04)$ & $+0.01 \pm 0.04( \pm 0.04)$ & $+0.03 \pm 0.03( \pm 0.04)$ & $+0.04 \pm 0.02( \pm 0.04)$ \\
{$[\mathrm{Cr} / \mathrm{Fe}]$} & $-0.11 \pm 0.21( \pm 0.05)$ & $+0.00 \pm 0.01( \pm 0.05)$ & $+0.04 \pm 0.03( \pm 0.05)$ & $+0.05 \pm 0.01( \pm 0.05)$ \\
{$[\mathrm{La} / \mathrm{Fe}]$} & $-0.14 \pm 0.07( \pm 0.04)$ & $+0.18 \pm 0.08( \pm 0.04)$ & $-0.08 \pm 0.04( \pm 0.04)$ & $-0.05 \pm 0.02( \pm 0.04)$ \\
{$[\mathrm{Mg} / \mathrm{Fe}]$} & $+0.33 \pm 0.10( \pm 0.09)$ & $+0.12 \pm 0.06( \pm 0.09)$ & $+0.10 \pm 0.08( \pm 0.09)$ & $+0.27 \pm 0.05( \pm 0.09)$ \\
{$[\mathrm{Na} / \mathrm{Fe}]$} & $-0.12 \pm 0.04( \pm 0.04)$ & $+0.01 \pm 0.07( \pm 0.04)$ & $+0.18 \pm 0.01( \pm 0.04)$ & $+0.25 \pm 0.06( \pm 0.04)$ \\
{$[\mathrm{Nd} / \mathrm{Fe}]$} & $+0.03 \pm 0.06( \pm 0.13)$ & $+0.34 \pm 0.09( \pm 0.13)$ & $+0.07 \pm 0.02( \pm 0.13)$ & $+0.04 \pm 0.05( \pm 0.13)$ \\
{$[\mathrm{Ni} / \mathrm{Fe}]$} & $-0.03 \pm 0.02( \pm 0.02)$ & $-0.01 \pm 0.03( \pm 0.02)$ & $+0.03 \pm 0.01( \pm 0.02)$ & $+0.02 \pm 0.02( \pm 0.02)$ \\
{$[\mathrm{O} / \mathrm{Fe}]$} & $+0.00 \pm 0.16( \pm 0.08)$ & $+0.03 \pm 0.04( \pm 0.08)$ & $-0.27 \pm 0.04( \pm 0.08)$ & $-0.11 \pm 0.03( \pm 0.08)$ \\
{$[\mathrm{Sc} / \mathrm{Fe}]$} & $+0.00 \pm 0.03( \pm 0.05)$ & $+0.03 \pm 0.05( \pm 0.05)$ & $-0.02 \pm 0.02( \pm 0.05)$ & $-0.04 \pm 0.05( \pm 0.05)$ \\
{$[\mathrm{Si} / \mathrm{Fe}]$} & $+0.14 \pm 0.05( \pm 0.04)$ & $+0.02 \pm 0.03( \pm 0.04)$ & $+0.09 \pm 0.01( \pm 0.04)$ & $+0.06 \pm 0.02( \pm 0.04)$ \\
{$[\mathrm{Ti} / \mathrm{Fe}]$} & $-0.08 \pm 0.07( \pm 0.03)$ & $-0.03 \pm 0.06( \pm 0.03)$ & $-0.09 \pm 0.04( \pm 0.03)$ & $-0.07 \pm 0.03( \pm 0.07)$ \\
{$[\mathrm{V} / \mathrm{Fe}]$} & $-0.08 \pm 0.05( \pm 0.06)$ & $+0.01 \pm 0.09( \pm 0.06)$ & $+0.04 \pm 0.05( \pm 0.06)$ & $+0.06 \pm 0.03( \pm 0.06)$ \\
{$[\mathrm{Y} / \mathrm{Fe}]$} & $-0.23 \pm 0.23( \pm 0.04)$ & $-0.03 \pm 0.08( \pm 0.04)$ & $-0.09 \pm 0.03( \pm 0.04)$ & $-0.11 \pm 0.01( \pm 0.04)$ \\
\hline
\end{tabular}


Table 8. High-resolution average Be 32 abundances.

\begin{tabular}{lccc}
\hline \hline & Here & $\mathrm{F} 10^{a}$ & $\mathrm{~B}^{b} 8^{b}$ \\
\hline$R=\lambda / \delta \lambda$ & 30000 & 28000 & 40000 \\
$S / N$ & $50-100$ & 100 & $45-100$ \\
\hline$[\mathrm{Fe} / \mathrm{H}]$ & $-0.30 \pm 0.02$ & $-0.30 \pm 0.02$ & $-0.29 \pm 0.04$ \\
{$[\mathrm{Al} / \mathrm{Fe}]$} & $+0.12 \pm 0.05$ & $+0.19 \pm 0.06$ & $+0.11 \pm 0.10$ \\
{$[\mathrm{Ba} / \mathrm{Fe}]$} & $+0.51 \pm 0.12$ & - & $+0.29 \pm 0.10$ \\
{$[\mathrm{Ca} / \mathrm{Fe}]$} & $-0.05 \pm 0.01$ & $-0.07 \pm 0.01$ & $+0.07 \pm 0.04$ \\
{$[\mathrm{Co} / \mathrm{Fe}]$} & $+0.07 \pm 0.05$ & $+0.00 \pm 0.01$ & - \\
{$[\mathrm{Cr} / \mathrm{Fe}]$} & $-0.11 \pm 0.21$ & $-0.16 \pm 0.11$ & $-0.05 \pm 0.04$ \\
{$[\mathrm{La} / \mathrm{Fe}]$} & $-0.14 \pm 0.07$ & - & - \\
{$[\mathrm{Mg} / \mathrm{Fe}]$} & $+0.33 \pm 0.10$ & $+0.13 \pm 0.01$ & $+0.27 \pm 0.08$ \\
{$[\mathrm{Na} / \mathrm{Fe}]$} & $-0.12 \pm 0.04$ & $+0.20 \pm 0.01$ & $+0.13 \pm 0.02$ \\
{$[\mathrm{Ni} / \mathrm{Fe}]$} & $-0.03 \pm 0.02$ & $-0.02 \pm 0.01$ & $+0.00 \pm 0.04$ \\
{$[\mathrm{O} / \mathrm{Fe}]$} & $+0.00 \pm 0.16$ & $-0.01 \pm 0.03$ & - \\
{$[\mathrm{Sc} / \mathrm{Fe}]$} & $+0.00 \pm 0.03$ & - & - \\
{$[\mathrm{Si} / \mathrm{Fe}]$} & $+0.14 \pm 0.05$ & $+0.27 \pm 0.05$ & $+0.12 \pm 0.04$ \\
{$[\mathrm{Ti} / \mathrm{Fe}]$} & $-0.08 \pm 0.07$ & $-0.17 \pm 0.01$ & $+0.11 \pm 0.06$ \\
{$[\mathrm{~V} / \mathrm{Fe}]$} & $-0.08 \pm 0.05$ & - & - \\
{$[\mathrm{Y} / \mathrm{Fe}]$} & $-0.23 \pm 0.23$ & - & - \\
\hline
\end{tabular}

Notes. ${ }^{(a)}$ Friel et al. (2010), from 2 stars. ${ }^{(b)}$ Bragaglia et al. (2008) and Sestito et al. (2006), from 10 red clump and RGB stars.

supersolar, while all other abundance ratios are solar, and our abundance ratios agree well with literature values.

The three late-type Hyades giants $(028,041$, and 070) have been widely studied (e.g., Luck \& Challener 1995; Boyarchuk et al. 2000; Schuler et al. 2006, 2009; Mishenina et al. 2006, 2007; Fulbright et al. 2007). In Table 9 we compiled available literature data. Our temperatures are slightly lower (by $\sim 100 \mathrm{~K}$ ) than the literature ones, whereas our values of $\log g$ and $v_{\mathrm{t}}$ are similar. These marginal differences appear to have no significant impact on the derived abundance ratios, which agree very well with literature ones. Exceptions are $\mathrm{Al}, \mathrm{Ba}$, and $\mathrm{O}$, which suffer from technical measurement problems (not strictly related to the Hyades cluster) and are discussed in Sects. 3.3 and 5.

\subsection{Praesepe (NGC 2632)}

The cluster popularly known as Praesepe or Beehive (also called M 44, NGC 2632 or Melotte 88; $\alpha_{2000}=08^{\mathrm{h}} 40^{\mathrm{m}} 24^{\mathrm{s}}$ and $\delta_{2000}=$ $\left.+19^{\circ} 40^{\prime} 00^{\prime \prime}\right)$, is an old OC ( 0.65 Gyr, see Table 3$)$ well known from the antiquity. It is located in the Cancer constellation at a distance of $\simeq 175 \mathrm{pc}$, computed from Hipparcos parallaxes.

Its metal content was derived with different methods (e.g., Friel \& Boesgaard 1992; Komarov \& Basak 1993; Claria et al. 1996; Hui-Bon-Hoa \& Alecian 1998; Burkhart \& Coupry 1998, 2000; Dias et al. 2002; Pace et al. 2008). In general, all the quoted studies obtained a metallicity either barely or definitely supersolar. Of these, the high-resolution abundance determinations were derived mainly for dwarfs or early-type giants (e.g., Friel \& Boesgaard 1992; Burkhart \& Coupry 1998; An et al. 2007; Pace et al. 2008). Surprisingly, to our knowledge, there are no recent high-resolution abundance determinations of latetype giants in this cluster.

Table 11 shows a comparison of our results with some of the most recent high-resolution studies. In general, the $[\mathrm{Fe} / \mathrm{H}]$ we derived in our late-type giants lies in-between those of Pace et al. (2008) and An et al. (2007), suggesting that the proposed dichotomy of literature values (barely supersolar versus definitely supersolar) should be interpreted rather as an above average uncertainty. This larger than usual uncertainty could naturally arise from the different spectral types and abundance analysis methods employed in the literature. The $[\mathrm{Fe} / \mathrm{H}]$ ratio derived by (Burkhart \& Coupry 1998), based on Am stars, is on average $\simeq 0.3$ dex larger than the values obtained in other works using different spectral-type stars. Although these stars should in principle reflect the chemical composition of the cluster, Am stars always have overabundant $\mathrm{Fe}$ abundances relative to other objects in the same clusters, without a clear explanation appearing in the literature. As in the case of the Hyades, $\mathrm{Na}$ and $\mathrm{O}$ abundances derived by us appear marginally discrepant with those by Pace et al. (2008), and will be discussed in more detail in Sect. 5.

\section{Discussion of abundance ratios}

As in Paper I, we compared our abundance ratios (and those from Paper I) with both others in the literature and the abundances of the Galactic disc field stars from Reddy et al. $(2006,2003)$ in Figs. 3 to 6. We extended the open cluster abundance compilation of Paper I (see Table 12) with both recent published works and old studies that were not included in the previous version. In both cases, as in Paper I, we included only studies based on high-resolution $(R \gtrsim 18000)$ spectroscopy. When more than one determination was available for one cluster, we simply plotted them all to give a realistic idea of the uncertainties involved in the compilation, and we did not attempt to correct for differences between the abundance analysis procedures $(\log g f$, solar reference, and so on), because this would be beyond the scope of the present article.

\subsection{Iron-peak element ratios}

Figure 3 shows the abundance ratios of iron-peak elements. Our OC with abundances close to solar (i.e., Hyades, Praesepe, and NGC 752) are in very good agreement with the results obtained in other OC studied with high-resolution spectra and in disc stars of similar metallicity. A larger scatter or marginal discrepancies are sometimes observed for the odd elements Sc, V, and Co, but this is because of the well-known hyperfine structure (HFS) of the lines usually employed in the analysis. The element that appears to suffer more from these effects is vanadium. This scatter is due, at least in part, to the different procedures used in the literature for treating the HFS splitting. We stress that in our case, we do not attempt any HFS correction.

The most metal-poor and oldest OC in our sample, Be 32, has a puzzling behaviour. While all its iron-peak abundance ratios are still compatible with the literature values for OC and field stars of similar metallicity (uncertainties are large), some underlying discrepancy could be present. For example, HFS should cause an overestimate (and not an underestimate) of vanadium. In addition, chromium appears to be lower than solar. We note that (see Table 8) the literature $\mathrm{Co}$ and $\mathrm{Cr}$ determinations by Friel et al. (2010), Sestito et al. (2006), and Bragaglia et al. (2008) are very similar to ours. In the case of our $[\mathrm{Cr} / \mathrm{Fe}]$ measurement for Be 32 , we must note that our two giants appear to exhibit quite different $[\mathrm{Cr} / \mathrm{Fe}]$ abundances, resulting in a large scatter in the cluster average value. This large scatter is most probably due to a measurement uncertainty, and should not be considered significant.

In Paper I, we noticed a peculiar behaviour in the Ni abundance ratios of literature OC determinations: they appear to be slightly richer in Ni than field stars by roughly 0.05 dex. Our $[\mathrm{Ni} / \mathrm{Fe}]$ ratios are in closer agreement with the field star determinations than with the OC ones. Although this difference is small (within the uncertainties), it appears systematic in nature, and 
R. Carrera and E. Pancino: Abundances of four open clusters

Table 9. Abundance comparison of individual Hyades stars (see text).

\begin{tabular}{|c|c|c|c|c|c|c|}
\hline Parameter & Here & S09/S06 & M07/M06 & F07 & Bo00 & LC95 \\
\hline Resolution & 30000 & 60000 & 42000 & 30000 & 45000 & 30000 \\
\hline$S / N$ & $300-600$ & $\sim 500$ & $100-350$ & 175 & $100-300$ & $>100$ \\
\hline Star & \multicolumn{6}{|c|}{$028(\gamma$ tau $)$} \\
\hline$T_{\text {eff }}(\mathrm{K})$ & 4750 & 4965 & 4955 & 4823 & 4956 & 4900 \\
\hline $\log g(\operatorname{dex})$ & 2.7 & 2.63 & 2.7 & 2.43 & 2.83 & 2.6 \\
\hline$v_{\mathrm{t}}\left(\mathrm{km} \mathrm{s}^{-1}\right)$ & 1.4 & 1.32 & 1.4 & 1.57 & 1.35 & 2.0 \\
\hline$[\mathrm{FeI} / \mathrm{H}]$ & $+0.12 \pm 0.01$ & $+0.14 \pm 0.08$ & +0.11 & $+0.16 \pm 0.05$ & $+0.11 \pm 0.01$ & $+0.13 \pm 0.02$ \\
\hline$[\mathrm{FeII} / \mathrm{H}]$ & $+0.13 \pm 0.03$ & $+0.22 \pm 0.16$ & +0.10 & $+0.09 \pm 0.08$ & $+0.11 \pm 0.02$ & $+0.12 \pm 0.02$ \\
\hline$[\mathrm{Al} / \mathrm{Fe}]$ & $-0.01 \pm 0.05$ & $+0.20 \pm 0.01$ & - & $+0.19 \pm 0.07$ & $+0.12 \pm 0.00$ & $-0.17 \pm 0.03$ \\
\hline$[\mathrm{Ba} / \mathrm{Fe}]$ & $+0.37 \pm 0.05$ & - & -0.07 & - & $+0.09 \pm 0.05$ & $-0.04 \pm 0.00$ \\
\hline$[\mathrm{Ca} / \mathrm{Fe}]$ & $-0.07 \pm 0.03$ & - & $+0.10 \pm 0.12$ & $-0.01 \pm 0.11$ & $+0.01 \pm 0.04$ & $-0.28 \pm 0.04$ \\
\hline$[\mathrm{Co} / \mathrm{Fe}]$ & $+0.00 \pm 0.04$ & - & - & - & $+0.02 \pm 0.02$ & $+0.04 \pm 0.04$ \\
\hline$[\mathrm{Cr} / \mathrm{Fe}]$ & $+0.02 \pm 0.03$ & - & - & - & $-0.01 \pm 0.02$ & $-0.03 \pm 0.11$ \\
\hline$[\mathrm{La} / \mathrm{Fe}]$ & $-0.12 \pm 0.06$ & - & -0.23 & - & $-0.03 \pm 0.02$ & - \\
\hline$[\mathrm{Mg} / \mathrm{Fe}]$ & $+0.13 \pm 0.05$ & $+0.43 \pm 0.01$ & -0.08 & $+0.03 \pm 0.07$ & $+0.16 \pm 0.02$ & $+0.18 \pm 0.05$ \\
\hline$[\mathrm{Na} / \mathrm{Fe}]$ & $+0.19 \pm 0.02$ & $+0.49 \pm 0.05$ & +0.22 & $+0.05 \pm 0.11$ & $+0.32 \pm 0.01$ & $+0.22 \pm 0.05$ \\
\hline$[\mathrm{Nd} / \mathrm{Fe}]$ & $+0.04 \pm 0.29$ & - & -0.15 & - & $-0.02 \pm 0.03$ & $+0.07 \pm 0.00$ \\
\hline$[\mathrm{Ni} / \mathrm{Fe}]$ & $+0.02 \pm 0.02$ & $+0.12 \pm 0.07$ & $-0.04 \pm 0.12$ & - & $+0.00 \pm 0.03$ & $+0.06 \pm 0.03$ \\
\hline$[\mathrm{O} / \mathrm{Fe}]$ & $-0.35 \pm 0.07$ & $-0.09 \pm 0.06$ & -0.09 & $-0.04 \pm 0.11$ & - & - \\
\hline$[\mathrm{Sc} / \mathrm{Fe}]$ & $-0.04 \pm 0.05$ & - & - & - & $+0.00 \pm 0.02$ & $+0.01 \pm 0.08$ \\
\hline$[\mathrm{Si} / \mathrm{Fe}]$ & $+0.09 \pm 0.03$ & - & $+0.07 \pm 0.12$ & $+0.09 \pm 0.09$ & $+0.09 \pm 0.03$ & $+0.21 \pm 0.03$ \\
\hline [Til/Fe] & $-0.12 \pm 0.02$ & - & - & $-0.05 \pm 0.10$ & $-0.01 \pm 0.01$ & $-0.14 \pm 0.03$ \\
\hline [TiII/Fe] & $-0.03 \pm 0.06$ & - & - & $-0.04 \pm 0.15$ & - & - \\
\hline$[\mathrm{V} / \mathrm{Fe}]$ & $+0.02 \pm 0.04$ & - & - & - & $+0.01 \pm 0.02$ & - \\
\hline$[\mathrm{Y} / \mathrm{Fe}]$ & $-0.12 \pm 0.05$ & - & -0.11 & - & $+0.01 \pm 0.01$ & $+0.10 \pm 0.00$ \\
\hline Star & \multicolumn{6}{|c|}{$041(\delta$ tau $)$} \\
\hline$T_{\mathrm{eff}}(\mathrm{K})$ & 4800 & 4938 & 4975 & - & 4980 & 4875 \\
\hline $\log g(\operatorname{dex})$ & 2.8 & 2.69 & 2.65 & - & 2.83 & 2.4 \\
\hline$v_{\mathrm{t}}\left(\mathrm{km} \mathrm{s}^{-1}\right)$ & 1.4 & 1.40 & 1.4 & - & 1.25 & 2.0 \\
\hline$[\mathrm{FeI} / \mathrm{H}]$ & $+0.10 \pm 0.01$ & $+0.14 \pm 0.07$ & +0.11 & - & $+0.19 \pm 0.01$ & $+0.07 \pm 0.01$ \\
\hline$[\mathrm{FeII} / \mathrm{H}]$ & $+0.13 \pm 0.03$ & $+0.26 \pm 0.16$ & +0.07 & 一 & $+0.18 \pm 0.03$ & $+0.04 \pm 0.02$ \\
\hline$[\mathrm{Al} / \mathrm{Fe}]$ & $+0.00 \pm 0.05$ & $+0.16 \pm 0.01$ & - & - & $+0.08 \pm 0.01$ & $-0.14 \pm 0.01$ \\
\hline$[\mathrm{Ba} / \mathrm{Fe}]$ & $+0.39 \pm 0.05$ & - & -0.02 & - & $+0.15 \pm 0.01$ & $-0.13 \pm 0.00$ \\
\hline$[\mathrm{Ca} / \mathrm{Fe}]$ & $-0.06 \pm 0.03$ & - & $+0.08 \pm 0.12$ & - & $+0.00 \pm 0.05$ & $-0.17 \pm 0.06$ \\
\hline$[\mathrm{Co} / \mathrm{Fe}]$ & $+0.01 \pm 0.03$ & - & - & - & $+0.01 \pm 0.03$ & $+0.10 \pm 0.05$ \\
\hline$[\mathrm{Cr} / \mathrm{Fe}]$ & $+0.03 \pm 0.03$ & - & - & - & $-0.04 \pm 0.02$ & $-0.06 \pm 0.09$ \\
\hline$[\mathrm{La} / \mathrm{Fe}]$ & $-0.08 \pm 0.05$ & - & -0.33 & - & $-0.05 \pm 0.09$ & - \\
\hline$[\mathrm{Mg} / \mathrm{Fe}]$ & $+0.06 \pm 0.04$ & $+0.36 \pm 0.02$ & -0.10 & - & $+0.15 \pm 0.01$ & $+0.33 \pm 0.07$ \\
\hline$[\mathrm{Na} / \mathrm{Fe}]$ & $+0.18 \pm 0.02$ & $+0.44 \pm 0.05$ & +0.16 & - & $+0.32 \pm 0.01$ & $+0.28 \pm 0.05$ \\
\hline$[\mathrm{Nd} / \mathrm{Fe}]$ & $+0.08 \pm 0.30$ & - & -0.17 & - & $+0.02 \pm 0.02$ & $-0.17 \pm 0.00$ \\
\hline$[\mathrm{Ni} / \mathrm{Fe}]$ & $+0.04 \pm 0.02$ & $-0.03 \pm 0.06$ & $+0.06 \pm 0.09$ & - & $+0.09 \pm 0.06$ & $+0.11 \pm 0.03$ \\
\hline$[\mathrm{O} / \mathrm{Fe}]$ & $-0.25 \pm 0.05$ & $-0.03 \pm 0.06$ & -0.21 & - & - & - \\
\hline$[\mathrm{Sc} / \mathrm{Fe}]$ & $+0.00 \pm 0.05$ & - & - & - & $+0.01 \pm 0.02$ & $-0.02 \pm 0.07$ \\
\hline$[\mathrm{Si} / \mathrm{Fe}]$ & $+0.09 \pm 0.02$ & - & $+0.07 \pm 0.11$ & - & $+0.06 \pm 0.02$ & $+0.23 \pm 0.03$ \\
\hline$[\mathrm{TiI} / \mathrm{Fe}]$ & $-0.11 \pm 0.02$ & - & - & - & $-0.04 \pm 0.02$ & $-0.07 \pm 0.03$ \\
\hline [TiII/Fe] & $-0.00 \pm 0.07$ & - & - & - & - & - \\
\hline$[\mathrm{V} / \mathrm{Fe}]$ & $+0.00 \pm 0.03$ & - & - & - & $+0.02 \pm 0.02$ & - \\
\hline$[\mathrm{Y} / \mathrm{Fe}]$ & $-0.06 \pm 0.06$ & - & -0.10 & - & $-0.04 \pm 0.03$ & $+0.19 \pm 0.00$ \\
\hline Star & \multicolumn{6}{|c|}{$070(\epsilon \mathrm{tau})$} \\
\hline$T_{\text {eff }}(\mathrm{K})$ & 4800 & 4911 & 4925 & 4838 & 4880 & - \\
\hline $\log g(\operatorname{dex})$ & 2.8 & 2.57 & 2.55 & 2.52 & 2.50 & - \\
\hline$v_{\mathrm{t}}\left(\mathrm{km} \mathrm{s}^{-1}\right)$ & 1.6 & 1.47 & 1.4 & 1.63 & 1.46 & - \\
\hline$[\mathrm{FeI} / \mathrm{H}]$ & $+0.11 \pm 0.01$ & $+0.20 \pm 0.08$ & +0.11 & $+0.21 \pm 0.07$ & $+0.11 \pm 0.01$ & - \\
\hline$[\mathrm{FeII} / \mathrm{H}]$ & $+0.09 \pm 0.03$ & $+0.22 \pm 0.16$ & +0.11 & $+0.18 \pm 0.10$ & $+0.05 \pm 0.03$ & - \\
\hline$[\mathrm{Al} / \mathrm{Fe}]$ & $+0.02 \pm 0.05$ & $+0.15 \pm 0.01$ & - & $+0.17 \pm 0.08$ & $+0.20 \pm 0.01$ & - \\
\hline$[\mathrm{Ba} / \mathrm{Fe}]$ & $+0.31 \pm 0.05$ & - & -0.02 & - & $+0.09 \pm 0.01$ & - \\
\hline$[\mathrm{Ca} / \mathrm{Fe}]$ & $-0.07 \pm 0.02$ & - & $+0.11 \pm 0.12$ & $+0.01 \pm 0.10$ & $+0.09 \pm 0.03$ & - \\
\hline$[\mathrm{Co} / \mathrm{Fe}]$ & $+0.06 \pm 0.03$ & - & - & - & $-0.01 \pm 0.04$ & - \\
\hline$[\mathrm{Cr} / \mathrm{Fe}]$ & $+0.08 \pm 0.04$ & - & - & - & $+0.00 \pm 0.01$ & - \\
\hline$[\mathrm{La} / \mathrm{Fe}]$ & $-0.05 \pm 0.05$ & - & -0.20 & - & $-0.17 \pm 0.00$ & - \\
\hline$[\mathrm{Mg} / \mathrm{Fe}]$ & $+0.21 \pm 0.07$ & $+0.37 \pm 0.02$ & -0.08 & $-0.03 \pm 0.08$ & - & - \\
\hline$[\mathrm{Na} / \mathrm{Fe}]$ & $+0.18 \pm 0.02$ & $+0.41 \pm 0.04$ & +0.23 & $+0.04 \pm 0.11$ & $+0.40 \pm 0.04$ & - \\
\hline$[\mathrm{Nd} / \mathrm{Fe}]$ & $+0.08 \pm 0.28$ & - & -0.21 & - & $-0.10 \pm 0.05$ & - \\
\hline$[\mathrm{Ni} / \mathrm{Fe}]$ & $+0.03 \pm 0.02$ & $+0.06 \pm 0.08$ & $+0.09 \pm 0.11$ & - & $+0.00 \pm 0.02$ & - \\
\hline$[\mathrm{O} / \mathrm{Fe}]$ & $-0.22 \pm 0.07$ & $-0.13 \pm 0.06$ & -0.01 & $-0.04 \pm 0.13$ & - & - \\
\hline$[\mathrm{Sc} / \mathrm{Fe}]$ & $-0.04 \pm 0.05$ & - & - & - & - & - \\
\hline$[\mathrm{Si} / \mathrm{Fe}]$ & $+0.10 \pm 0.03$ & - & $+0.09 \pm 0.11$ & $+0.05 \pm 0.11$ & $+0.09 \pm 0.03$ & - \\
\hline$[\mathrm{TiI} / \mathrm{Fe}]$ & $-0.06 \pm 0.01$ & - & - & $-0.01 \pm 0.08$ & $-0.05 \pm 0.03$ & - \\
\hline [Till/Fe] & $-0.02 \pm 0.11$ & - & - & $-0.14 \pm 0.11$ & - & - \\
\hline$[\mathrm{V} / \mathrm{Fe}]$ & $+0.09 \pm 0.03$ & - & - & - & $-0.04 \pm 0.03$ & - \\
\hline$[\mathrm{Y} / \mathrm{Fe}]$ & $-0.07 \pm 0.05$ & - & -0.11 & - & $-0.05 \pm 0.03$ & - \\
\hline
\end{tabular}


Table 10. High-resolution average Hyades abundances from giants.

\begin{tabular}{lcccc}
\hline \hline & Here & S09/S06 $^{a}$ & Bo00 $^{b}$ & V99 $^{c}$ \\
\hline$R=\lambda / \delta \lambda$ & 30000 & 60000 & 45000 & $30-65000$ \\
$S / N$ & $50-100$ & $100-200$ & $100-300$ & $\sim 200$ \\
\hline$[\mathrm{Fe} / \mathrm{H}]$ & $+0.11 \pm 0.01$ & $+0.21 \pm 0.04$ & $+0.12 \pm 0.06$ & $-0.05 \pm 0.03$ \\
{$[\mathrm{Al} / \mathrm{Fe}]$} & $+0.00 \pm 0.02$ & $+0.17 \pm 0.03$ & $+0.13 \pm 0.06$ & - \\
{$[\mathrm{Ba} / \mathrm{Fe}]$} & $+0.36 \pm 0.04$ & - & $+0.10 \pm 0.03$ & $-0.03 \pm 0.07$ \\
{$[\mathrm{Ca} / \mathrm{Fe}]$} & $-0.07 \pm 0.01$ & - & $+0.03 \pm 0.05$ & $+0.03 \pm 0.04$ \\
{$[\mathrm{Co} / \mathrm{Fe}]$} & $+0.03 \pm 0.03$ & - & $+0.00 \pm 0.02$ & - \\
{$[\mathrm{Cr} / \mathrm{Fe}]$} & $+0.04 \pm 0.03$ & - & $-0.02 \pm 0.02$ & - \\
{$[\mathrm{La} / \mathrm{Fe}]$} & $-0.08 \pm 0.04$ & - & $-0.10 \pm 0.08$ & - \\
{$[\mathrm{Mg} / \mathrm{Fe}]$} & $+0.10 \pm 0.08$ & $+0.38 \pm 0.03$ & $+0.16 \pm 0.01$ & $+0.17 \pm 0.04$ \\
{$[\mathrm{Na} / \mathrm{Fe}]$} & $+0.18 \pm 0.01$ & $+0.45 \pm 0.04$ & $+0.37 \pm 0.05$ & $+0.10 \pm 0.05$ \\
{$[\mathrm{Ni} / \mathrm{Fe}]$} & $+0.03 \pm 0.01$ & $+0.05 \pm 0.07$ & $+0.05 \pm 0.06$ & $+0.04 \pm 0.04$ \\
{$[\mathrm{O} / \mathrm{Fe}]$} & $-0.27 \pm 0.04$ & $-0.08 \pm 0.05$ & - & $+0.11 \pm 0.04$ \\
{$[\mathrm{Sc} / \mathrm{Fe}]$} & $-0.02 \pm 0.02$ & - & $+0.00 \pm 0.01$ & $+0.03 \pm 0.07$ \\
{$[\mathrm{Si} / \mathrm{Fe}]$} & $+0.09 \pm 0.01$ & - & $+0.07 \pm 0.01$ & $+0.13 \pm 0.03$ \\
{$[\mathrm{Ti} / \mathrm{Fe}]$} & $-0.09 \pm 0.04$ & - & $-0.04 \pm 0.02$ & - \\
{$[\mathrm{V} / \mathrm{Fe}]$} & $+0.04 \pm 0.05$ & - & $-0.01 \pm 0.03$ & - \\
{$[\mathrm{Y} / \mathrm{Fe}]$} & $-0.09 \pm 0.03$ & - & $-0.04 \pm 0.02$ & $-0.04 \pm 0.07$ \\
\hline
\end{tabular}

References. ${ }^{(a)}$ Schuler et al. (2009) and Schuler et al. (2006), from the same $3 \mathrm{~K}$ giants studied here. ${ }^{(b)}$ Boyarchuk et al. (2000), from the same $3 \mathrm{~K}$ giants studied here. ${ }^{(c)}$ Varenne \& Monier (1999), from $29 \mathrm{~F}$ dwarfs.

Table 11. High-resolution average Praesepe (NGC 2632) abundances.

\begin{tabular}{lcccc}
\hline \hline & Here & P08 $^{a}$ & ${\mathrm{~A} 07^{b}}^{b}$ & ${\mathrm{Bu} 98^{c}}^{c}$ \\
\hline$R=\lambda / \delta \lambda$ & 30000 & 100000 & 55000 & 90000 \\
$S / N$ & $50-100$ & $\simeq 80$ & $\simeq 100$ & $\sim 200$ \\
\hline$[\mathrm{Fe} / \mathrm{H}]$ & $+0.16 \pm 0.05$ & $+0.27 \pm 0.10$ & $+0.11 \pm 0.03$ & $+0.40 \pm 0.14$ \\
{$[\mathrm{Al} / \mathrm{Fe}]$} & $+0.00 \pm 0.03$ & $-0.05 \pm 0.12$ & - & $-0.19 \pm 0.17$ \\
{$[\mathrm{Ba} / \mathrm{Fe}]$} & $+0.33 \pm 0.05$ & $+0.22 \pm 0.06$ & - & - \\
{$[\mathrm{Ca} / \mathrm{Fe}]$} & $-0.08 \pm 0.02$ & $+0.00 \pm 0.11$ & - & $+0.04 \pm 0.16$ \\
{$[\mathrm{Co} / \mathrm{Fe}]$} & $+0.04 \pm 0.02$ & - & - & - \\
{$[\mathrm{Cr} / \mathrm{Fe}]$} & $+0.05 \pm 0.01$ & $-0.01 \pm 0.08$ & - & - \\
{$[\mathrm{La} / \mathrm{Fe}]$} & $-0.05 \pm 0.02$ & - & - & - \\
{$[\mathrm{Mg} / \mathrm{Fe}]$} & $+0.27 \pm 0.05$ & - & - & - \\
{$[\mathrm{Na} / \mathrm{Fe}]$} & $+0.25 \pm 0.06$ & $-0.04 \pm 0.12$ & - & - \\
{$[\mathrm{Ni} / \mathrm{Fe}]$} & $+0.02 \pm 0.02$ & $-0.02 \pm 0.12$ & - & $+0.21 \pm 0.17$ \\
{$[\mathrm{O} / \mathrm{Fe}]$} & $-0.11 \pm 0.03$ & $-0.40 \pm 0.20$ & - & - \\
{$[\mathrm{Sc} / \mathrm{Fe}]$} & $-0.04 \pm 0.05$ & - & - & - \\
{$[\mathrm{Si} / \mathrm{Fe}]$} & $+0.06 \pm 0.02$ & $-0.01 \pm 0.12$ & - & - \\
{$[\mathrm{Ti} / \mathrm{Fe}]$} & $-0.07 \pm 0.03$ & $-0.04 \pm 0.12$ & - & - \\
{$[\mathrm{V} / \mathrm{Fe}]$} & $+0.06 \pm 0.03$ & - & - & - \\
{$[\mathrm{Y} / \mathrm{Fe}]$} & $-0.11 \pm 0.01$ & - & - & - \\
\hline
\end{tabular}

Notes. ${ }^{(a)}$ Pace et al. (2008), from $6 \mathrm{G}$ and $1 \mathrm{~F}$ main sequence stars. ${ }^{(b)}$ An et al. (2007), from $4 \mathrm{G}$ dwarfs stars. ${ }^{(c)}$ Burkhart \& Coupry (1998), from 10 Am stars.

we were unable to find any easy explanation, such as the choice of either solar reference abundances or the $\log g f$ system, of this discrepancy.

\subsection{Alpha-element ratios}

Figure 4 shows the abundance ratios of $\alpha$-elements. As for ironpeak elements, our measurements are always compatible with the literature values, within their uncertainties. Generally speaking, all our OC show roughly solar $\alpha$-enhancements, even Be 32, which has a lower metallicity.

However, some elements deserve some more discussion, as was noted in Paper I. For example, the $\log g f$ of calcium are quite uncertain, and we chose the VALD reference atomic data,

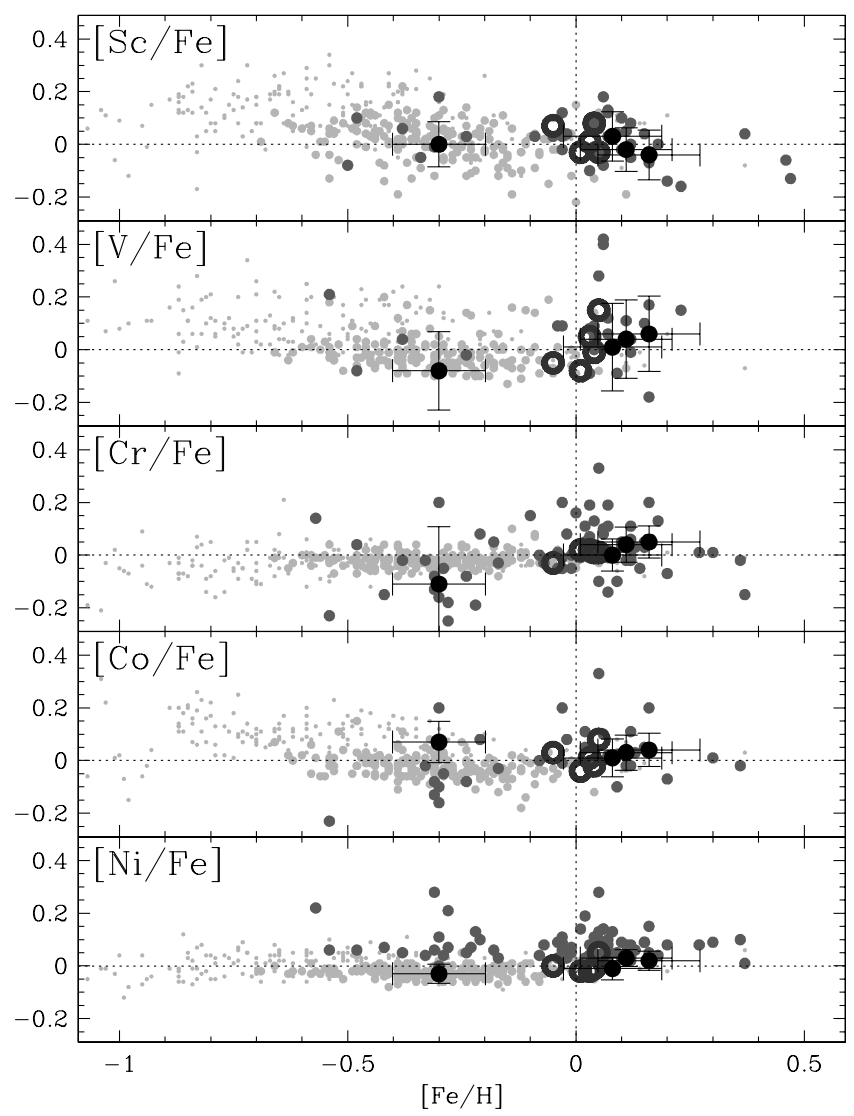

Fig. 3. Comparison between our iron-peak abundance ratios (large black dots), those of Paper I (large black open circles), high-resolution measurements listed in Table 12 (large dark grey dots), field stars belonging to the thin disc (light grey dots, Reddy et al. 2003), and to the thick disc (tiny light grey dots, Reddy et al. 2006). Errorbars in our measurements are the quadratic sum of all uncertainties discussed in Sects. 3.2 and 3.3.

which explains why our $[\mathrm{Ca} / \mathrm{Fe}]$ ratios are slightly lower than the bulk of literature determinations for cluster and disc stars. A similar problem affects the $\mathrm{Mg}$ lines, as can clearly be appreciated from the large spread of literature values. Our $[\mathrm{Mg} / \mathrm{Fe}] \mathrm{de}-$ terminations tend to lie on the upper envelope of literature ratios for OC. A deeper discussion of $\mathrm{Mg}$ abundances can be found in Paper I.

In the case of oxygen, the problem is instead in the difficulty in measuring its small lines. The forbidden [O I] line at $6300 \AA$, which we used in this paper, suffers from contamination by a Ni line and by telluric absorption features, while the O triplet around $7770 \AA$ (used by some other studies) suffers from NLTE effects. This is reflected by the large scatter in literature values.

\subsection{Heavy element ratios}

We determined abundances for three heavy s-process elements: $\mathrm{Ba}, \mathrm{La}$, and $\mathrm{Nd}$; and one light s-process element: Y (Fig. 5). Literature determinations for these elements are not numerous. D'Orazi et al. (2009) measured Ba in several OC using spectral synthesis to take into account HFS. The [Ba/Fe] abundances derived by D'Orazi et al. (2009) taking into account HFS do not differ significantly from other literature determinations (including ours). The $[\mathrm{Ba} / \mathrm{Fe}]$ ratios are clearly above solar for most of the clusters and they show a scatter larger than 0.5 . D'Orazi et al. (2009) found this scatter to be due to 


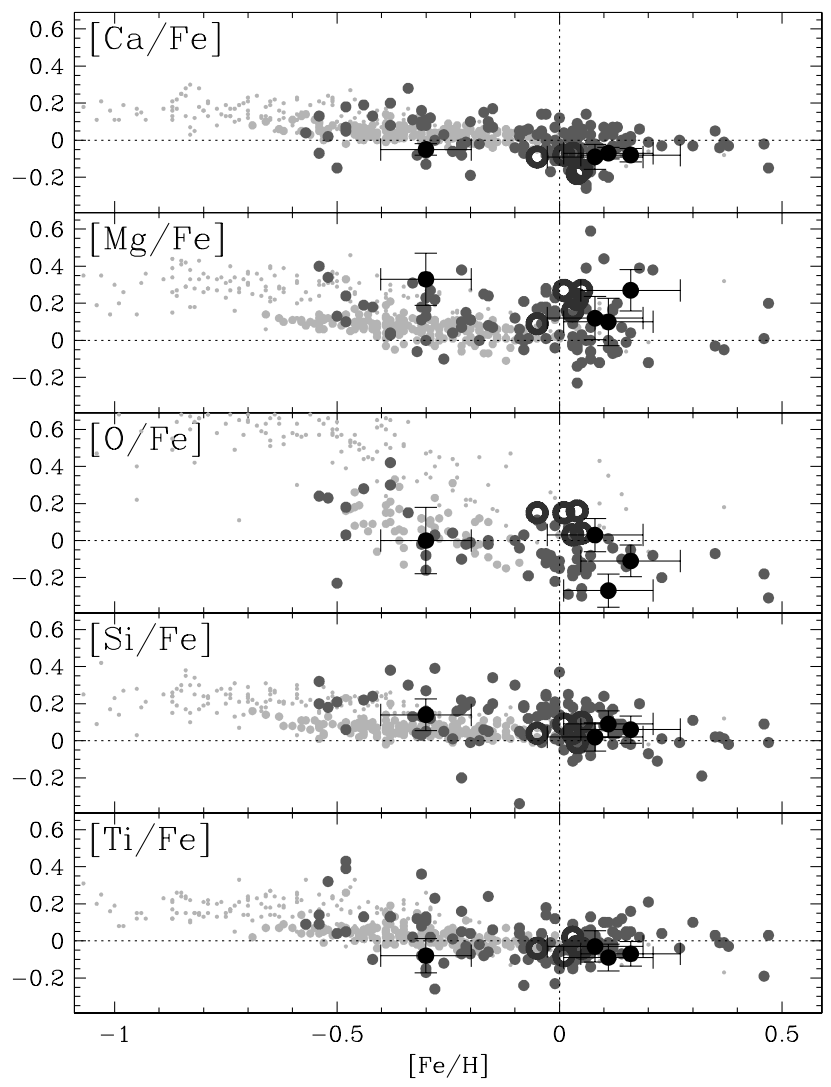

Fig. 4. Comparison of our $\alpha$-elements ratios with literature values. Symbols are the same as in Fig. 3.

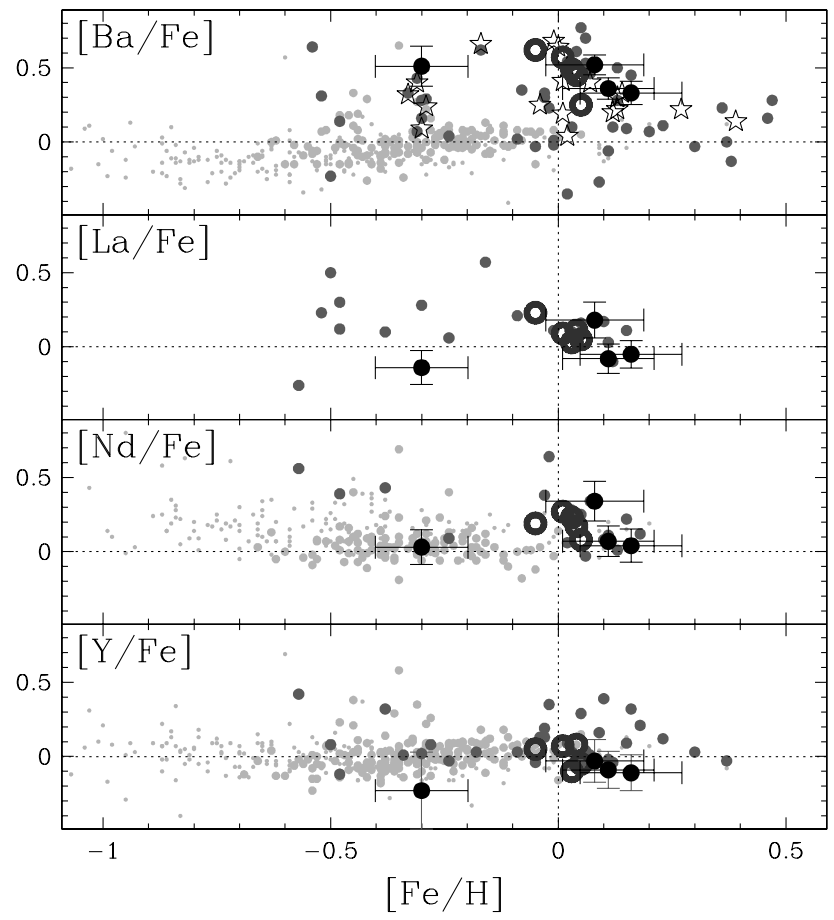

Fig. 5. Comparison of our s-process elements ratios with the literature ones. Symbols are the same as in Fig. 3, except for the black starlike symbols in the top $[\mathrm{Ba} / \mathrm{Fe}]$ panel, which represent the revision of $\mathrm{Ba}$ abundances with spectral synthesis performed by D'Orazi et al. (2009).

age: the Ba content appears to increase for younger clusters. The available lanthanum and neodymium lines were unfortunately

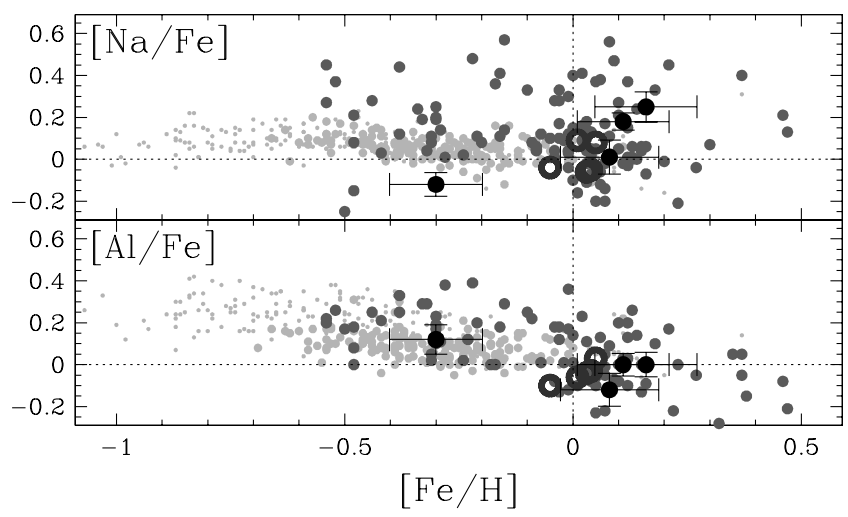

Fig. 6. Comparison between our $[\mathrm{Na} / \mathrm{Fe}]$ and $[\mathrm{Al} / \mathrm{Fe}]$ ratios and the literature ones. Symbols are the same as in Fig. 3.

relatively small, and we were able to find fewer published studies to compare with. As a result, the solar clusters (Hyades, Praesepe, and NGC 752) have La and Nd ratios in good agreement with the literature, while Be 32 appears to have lower $[\mathrm{La} / \mathrm{Fe}]$ and $[\mathrm{Nd} / \mathrm{Fe}]$ than the few studied $\mathrm{OC}$ at a similar metallicity, which are Mel 66 (Gratton \& Contarini 1994) and NGC 2243 (Smith \& Suntzeff 1987). However, our [Nd/Fe] agrees well with the field star solar ratios. The only light s-process element we could measure, Y, relies on a couple of weak lines that provide uncertain abundances (see the large errorbar in Fig. 5). Our Y ratio appears to be lower than all literature estimates, although still compatible with the solar values of field stars of similar metallicity, within the large uncertainties.

In summary, we can say that all the studied clusters appear to have roughly solar s-process enhancements, but it would be extremely interesting to attempt a more detailed study of s-process elements in OC, as done by D'Orazi et al. (2009) for barium.

\subsection{Ratios of $\mathrm{Na}$ and $\mathrm{Al}$ and anticorrelations}

As discussed in Paper I, the study of light elements in OC is quite interesting. The elements $\mathrm{Al}$ and $\mathrm{Na}$, together with $\mathrm{Mg}$, $\mathrm{O}, \mathrm{C}$, and $\mathrm{N}$, show puzzling (anti-)correlations in almost all of the studied globular clusters, in the Milky Way (see, e.g., Carretta et al. 2010; Pancino et al. 2010b, and references therein) and outside (e.g., Mucciarelli et al. 2009; Letarte et al. 2006). No (anti-)correlations were observed in either field stars (but see Martell \& Grebel 2010) or OC (Martell \& Smith 2009; de Silva et al. 2009; Smiljanic et al. 2009; and Paper I) so far. This suggests that metallicity, cluster size and age, or the environment must play a rôle, and therefore finding (anti-)correlations in some OC would be of enormous importance to put tighter constraints on the phenomenon.

We determined abundances of $\mathrm{Al}$ and $\mathrm{Na}$ and compared them with published results in Fig. 6. While in the case of aluminium the agreement with literature values is good, we find a significantly lower $[\mathrm{Na} / \mathrm{Fe}]$ ratio for Be 32 than for other clusters or field stars of similar metallicity. Generally speaking, the large scatter in $\mathrm{Na}$ determinations could be due to the difficulties in measuring $\mathrm{Na}$ lines, often affected by NLTE effects (Gratton et al. 1999), although no such scatter is observed among field stars. However, a few clusters have $[\mathrm{Na} / \mathrm{Fe}]$ lower than our Be 32 determination, and NLTE corrections (Gratton et al. 1999) could make the discrepancy of our Be $32 \mathrm{Na}$ determination even worse. Unfortunately, given the large scatter and the difficulty of 

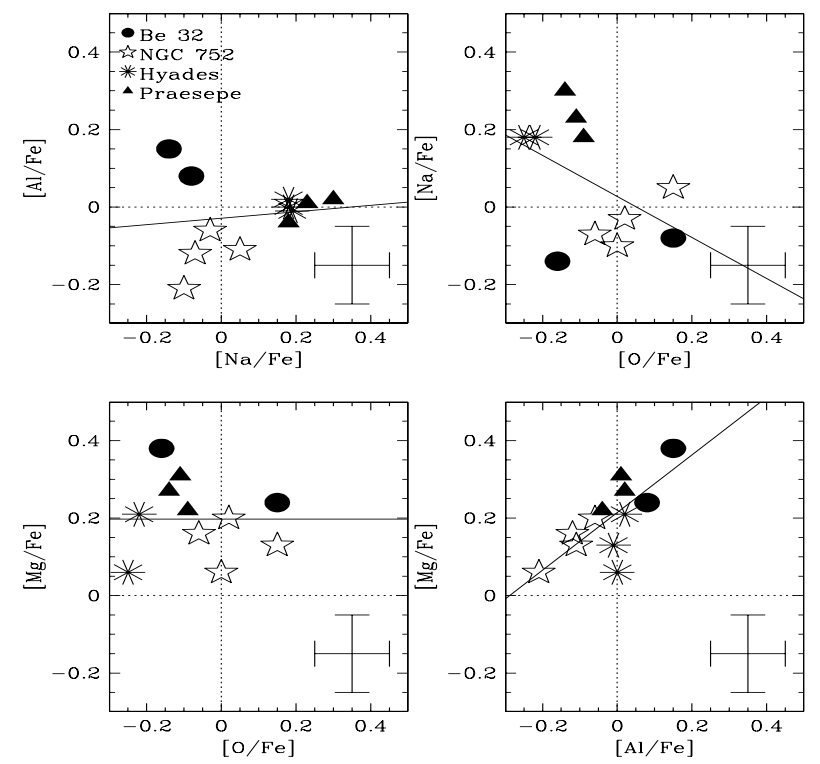

Fig. 7. A search for (anti)-correlations of $\mathrm{Al}, \mathrm{Mg}, \mathrm{Na}$, and $\mathrm{O}$ among our target stars. The four panels show different planes of abundance ratios, where stars belonging to each cluster are marked with different symbols. Dotted lines show solar values, solid lines show linear regressions and the typical uncertainty $(\sim 0.1 \mathrm{dex})$ is marked at the lower right corner of each panel.

measurement, it is difficult to either confirm or exclude the presence of some (small) intrinsic $[\mathrm{Na} / \mathrm{Fe}]$ scatter in this clusters.

In Fig. 7, all the studied stars occupy the "normal stars" loci, which is around solar for $\mathrm{Na}$ and $\mathrm{Al}$, and slightly $\alpha$-enhanced for $\mathrm{O}$ and $\mathrm{Mg}$ (see Sect. 5.2). There is a hint of correlation between $[\mathrm{Al} / \mathrm{Fe}]$ and $[\mathrm{Na} / \mathrm{Fe}]$, which was also observed for objects studied in Paper I. Of course, small variations in $T_{\text {eff }}$ could induce artificial correlations between element pairs, so the observed trend is most probably not-significant. However, we again note that the Na spread is very large (see above), suggesting that a small degree of chemical anomalies (barely hidden within the present observational uncertainties) cannot be completely excluded.

\section{Galactic trends}

The existence of trends in the chemical abundances with Galactocentric distance, $R_{\mathrm{gc}}$, vertical distance to the Galactic plane, $z$, and age, are key to understanding Galactic disc formation and evolution because they provide fundamental constraints on chemical evolution models. Different tracers have been used to investigate trends in the Galactic disc: OB stars (e.g., Daflon \& Cunha 2004), Cepheids (e.g., Lemasle et al. 2008), H II regions (e.g., Deharveng et al. 2000), and planetary nebulae (e.g., Costa et al. 2004). However, as coeval groups of stars at the same distance and with a homogeneous chemical composition, OC are the ideal test particles to investigate the existence of radial and vertical gradients and of an age-metallicity relation in the disc.

We complement the small sample of abundance ratios obtained here and in Paper I with a revised version of the literature data first presented in Paper I (Table 12). When a cluster had two or more abundance determinations available in the literature, we averaged them to make the figures easily readable and the error bars are, simply, calculated as the standard deviation. For those clusters with only one abundance determination, the error bars are the uncertainties in those determinations.
The heliocentric distances compiled in the updated version of the Dias et al. (2002) database were used to obtain $R_{\mathrm{gc}}$ and $z$ for each cluster, assuming $R_{\mathrm{GC}_{\odot}}=8.5 \mathrm{kpc}$. Ages were obtained from the same source, which is a compilation of different values available in the literature, hence might still be quite inaccurate for some clusters. In spite of its heterogeneity, our compilation contains a total of 89 clusters and is, to the best of our knowledge, the largest available in the literature, based on high-resolution spectroscopic abundances. Any attempt to homogenize this sample, for which abundances, distances, and ages have been derived from very different techniques, is clearly beyond the scope of this paper. This prevents us from a detailed analysis of the Galactic trends of all elements. For this reason, we focus only on $[\mathrm{Fe} / \mathrm{H}]$ and $[\alpha / \mathrm{Fe}]$ ratios. In spite of this heterogeneity, this analysis is still very useful owing to the number of clusters, and the large range of ages, and vertical and radial distances covered, even if the heterogeneity of the sample forces us to be extremely cautions when drawing any conclusion from the data.

\subsection{Trends with Galactocentric radius}

Radial gradients may arise when the disc forms, and different mechanisms can produce them: for example, different timescales of star formation at different distances (e.g., Schaye 2004); a radial variation in the infall of gas; or a change in the yield as a function of the radius (e.g., Molla et al. 1996). This initial radial gradient can be either amplified (steepened) or washed out (flattened) with time by radial mixing (e.g., Roškar et al. 2008).

Since the pioneering work of Janes (1979), OC have been widely used to investigate the gradient in metallicity with radius in the Galactic disc (e.g., Twarog et al. 2003; Friel et al. 2002, 2010; Magrini et al. 2009; Jacobson et al. 2011a,b). Friel (1995) reviewed the firsts investigations in this field. Since then, a great effort have been performed to obtain both homogeneous (e.g., Friel et al. 2002, 2010; Sestito et al. 2008) and/or larger samples (e.g., Twarog et al. 1997; Jacobson et al. 2011a,b). All these investigations agree on the fact that the iron content decreases with increasing radius (e.g., Friel et al. 2002). This behaviour has been generally considered linear with a slope between -0.05 and -0.09 dex $\mathrm{kpc}^{-1}$, depending on the cluster sample used. Similar trends were obtained for other different tracers of the disc (e.g., Andrievsky et al. 2004; Lemasle et al. 2008). Most of these works were limited to the inner $R_{\mathrm{gc}} \simeq 15 \mathrm{kpc}$. However, investigations based on samples containing clusters at larger distances (e.g., Twarog et al. 1997; Yong et al. 2005; Sestito et al. 2008 ) found that the $[\mathrm{Fe} / \mathrm{H}]$ ratio decreases as a function of increasing radius to $R_{\mathrm{gc}} \simeq 12.5 \mathrm{kpc}$ and appears to flatten from there outwards.

The variation in $[\mathrm{Fe} / \mathrm{H}]$ with $R_{\mathrm{gc}}$ in our compilation has been plotted in the top panel of Fig. 8. The whole sample is well fitted by a line with a slope of $-0.046 \pm 0.005 \mathrm{dex} \mathrm{kpc}^{-1}$ (long-dashed line), in concordance with the result obtained in Paper I from $\mathrm{a} \simeq 20 \%$ smaller sample $\left(-0.05 \pm 0.01 \mathrm{dex} \mathrm{kpc}^{-1}\right)$ and in other investigations in the literature (e.g., $-0.06 \pm 0.02 \mathrm{dex} \mathrm{kpc}{ }^{-1}$; Friel et al. 2002). The sample used here contains more clusters with distances larger than $R_{\mathrm{gc}} \geq 12 \mathrm{kpc}$. This allows us to investigate the discontinuity observed by some authors at $R_{\mathrm{gc}} \simeq$ $12-13 \mathrm{kpc}$. At first sight, no clear discontinuity in slope appears, partly because of the large range of $[\mathrm{Fe} / \mathrm{H}]$ at this radius $(\simeq 0.5$ dex $)$ and partly as a possible consequence of the heterogeneity of our sample. However, when we fit separately clusters inwards and outwards of $12.5 \mathrm{kpc}$, we find two significantly different slopes: the metallicity in the inner disc decreases with a slope of $-0.07 \pm 0.01 \mathrm{dex} \mathrm{kpc}^{-1}$, while in the outer disc the 


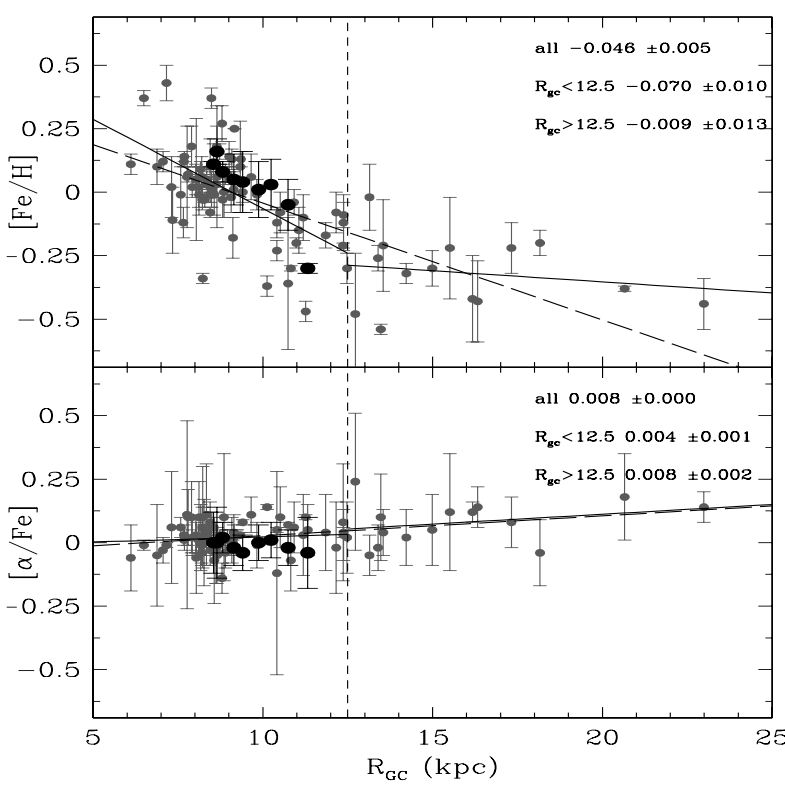

Fig. 8. Trends of $[\mathrm{Fe} / \mathrm{H}]$ (top panel) and $[\alpha / \mathrm{Fe}]$ (bottom panel) with galactocentric radius. Grey dots are OC compiled in Table 12, while black dots are the ones analysed here and in Paper I. A global linear fit is drawn in both panels (long-dashed line). Two separate linear fits of OC inside and outside $12.5 \mathrm{kpc}$ are also shown (solid lines).

slope is $-0.01 \pm 0.01 \mathrm{dex} \mathrm{kpc}{ }^{-1}$. The obtained slopes change within the uncertainties if the cut radius varies between 11.5 and $13.5 \mathrm{kpc}$. This is also in very good agreement with the recent results by Andreuzzi et al. (2011), who find $-0.07 \mathrm{dex} \mathrm{kpc}^{-1}$ in the inner $12 \mathrm{kpc}$. This bimodal behaviour can be explained by a different chemical enrichment and star formation in the inner and outer disc; (e.g., Chiappini et al. 2001; Magrini et al. 2009) however, a sharp discontinuity between the inner and outer disc is not expected theoretically.

The ratio $[\alpha / \mathrm{Fe}]$ reflects the relative contributions of type Ia and II supernovae: chemical evolution models predict an increase of this ratio with $R_{\mathrm{gc}}$ (e.g., Chiappini et al. 2001; Magrini et al. 2009). This tendency was indeed observed in OC by, e.g., Yong et al. (2005), Magrini et al. (2009), and in Paper I. The bottom panel of Fig. 8 shows the variation in $[\alpha / \mathrm{Fe}]$ with $R_{\mathrm{gc}}$ for our compilation: a weak increase in $\alpha$-element abundances with radius is apparent. However, the slope is still compatible with a flat distribution at the $1 \sigma$ level, as in Paper I, especially if the two outermost clusters are removed. The discontinuity observed for $[\mathrm{Fe} / \mathrm{H}]$ is not evident at all in $[\alpha / \mathrm{Fe}]$.

An accretion of a satellite into the outer disc could also explain the trend observed (e.g., Chiappini et al. 2001; Yong et al. 2005). In this case, we would expect to find some inhomogeneities corresponding to the trajectory of the merger. Carraro \& Bensby (2009) indeed found evidence that two OC, Berkeley 29 and Saurer 1, are related to the Sagittarius dwarf galaxy. Our compiled sample unfortunately do not allow us to investigate this question in depth.

\subsection{Time evolution of the radial gradient}

Chemical evolution models of the Galactic disc predict a variation in the metallicity gradient with time, but they disagree about the direction of this gradient variation (see Maciel et al. 2007, for a recent review), some predicting a steepening and some a

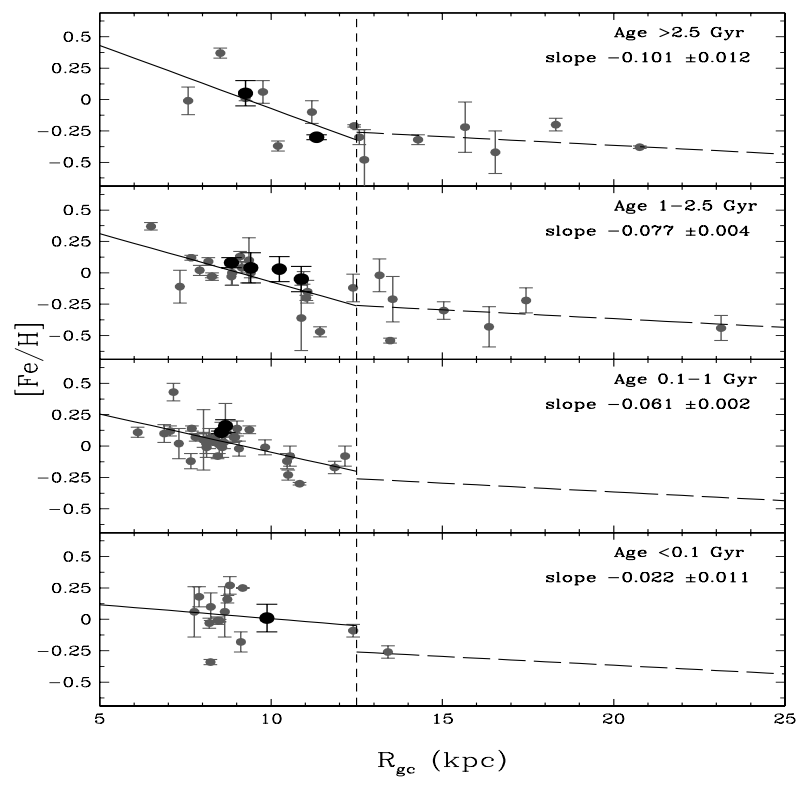

Fig. 9. Gradient in $[\mathrm{Fe} / \mathrm{H}]$ as a function of $R_{\mathrm{gc}}$ in four different age bins (labeled in top-right corner). A linear fit is performed for the OC within a radius of $R_{\mathrm{gc}}=12.5 \mathrm{kpc}$, and the slope indicated on each panel. A flatter and roughly constant slope is found outside a radius of $R_{\mathrm{gc}}=12.5 \mathrm{kpc}$.

flattening of the gradient with time. Studies based on metallicities derived from low-resolution spectroscopy found that old OC ( $\gtrsim 1 \mathrm{Gyr}$ ) followed a steeper radial gradient, $\sim-0.08 \mathrm{dex} \mathrm{kpc}^{-1}$, than the younger ones, $\sim-0.02 \mathrm{dex} \mathrm{kpc}^{-1}$ (Friel et al. 2002; Chen et al. 2003). Only recently have chemical abundances been derived from high-resolution spectroscopy for a sufficient number of OC to significantly investigate the variation in the radial gradient with time. As for studies based on low-resolution spectra, they agree that the gradient was steeper in the past and has flattened with time (Magrini et al. 2009; Andreuzzi et al. 2011). For example, on the basis of a sample of $\sim 70$ OC Andreuzzi et al. (2011) found that all objects younger than 4 Gyr display a similar gradient with a slope $-0.07 \mathrm{dex} \mathrm{kpc}^{-1}$ in the inner $12 \mathrm{kpc}$, while the one for older objects is steeper, $-0.15 \mathrm{dex} \mathrm{kpc}^{-1}$.

Other tracers have been used to study the time variation in radial gradients. Studies based on planetary nebulae found more puzzling results: while Maciel et al. (2003) found a flattening of the gradient with time, as generally observed for OC, Stanghellini \& Haywood (2010) found that the gradient steepens with time. At the moment, there is no explanation of this contradictory result. Comparisons among the slopes of the radial gradients described by populations of different ages also show that the gradient has flattened out in the past few Gyr (see Maciel \& Costa 2009, for a recent review).

To investigate the behaviour of the radial gradient in our compiled sample of high-resolution abundances, we plotted in Fig. 9 the gradient in $[\mathrm{Fe} / \mathrm{H}]$ as a function of $R_{\mathrm{gc}}$ in four different age bins. We obtained a linear fit in each age bin for the inner $12.5 \mathrm{kpc}$, and for the outer range we simply used the same fit as in Fig. 8, owing to the paucity of OC after age binning in this region. We found that the slope of the $[\mathrm{Fe} / \mathrm{H}]$ gradient increases as we go back in time from $-0.02 \pm 0.01 \mathrm{dex} \mathrm{kpc}^{-1}$ for objects younger than 0.1 Gyr to $-0.10 \pm 0.01 \mathrm{dex} \mathrm{kpc}^{-1}$ for clusters older than 2.5 Gyr. 


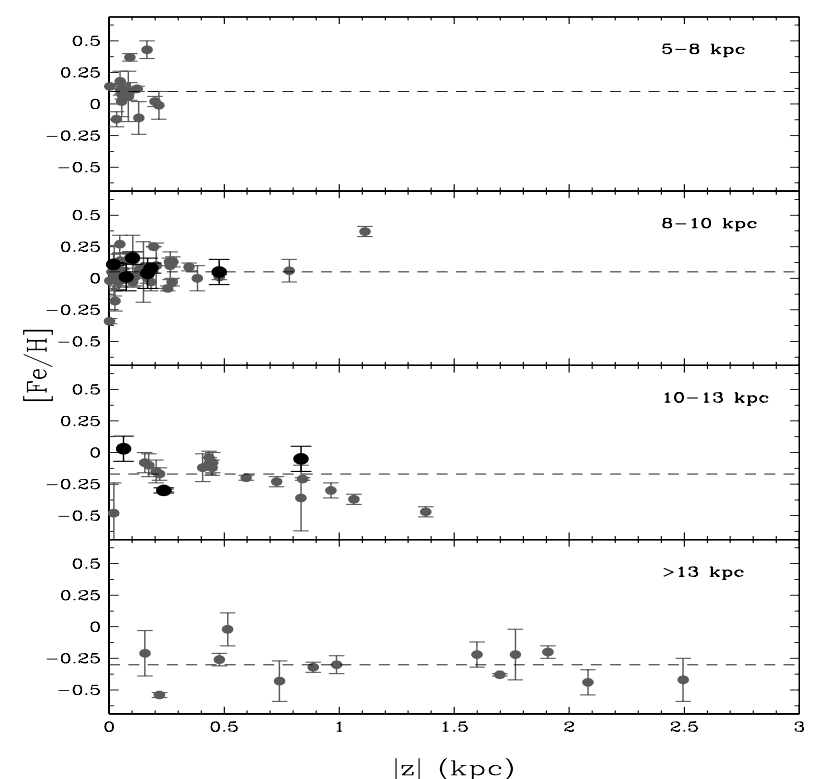

Fig. 10. Trends in $[\mathrm{Fe} / \mathrm{H}]$ with $|z|$ in four radial annuli as indicated on the top-right corner of each panel, moving outwards from the top to the bottom panel. Symbols are the same as in Fig. 8. As a reference, we plotted dashed lines in each panel, representing the median metallicity of clusters in each radial annulus.

\subsection{Trends with the disc scale-height}

Another interesting trend that could be investigated is the behaviour of $[\mathrm{Fe} / \mathrm{H}]$ with the vertical scale-height of the disc $z$, i.e., the vertical $[\mathrm{Fe} / \mathrm{H}]$ gradient. Although the formation of the thick discs remains an open question, the existence of vertical gradients can help us to discriminate among the mechanisms proposed to their formation. No vertical chemical gradients are expected in thick discs formed by heating caused by accretion events or major mergers. In contrast, vertical gradients may exist in discs thickened by gradual heating of the thin disc or before the gas has settled to form a thin disc (see Mould 2005, for a review). Up to now, there is no conclusive agreement about the existence of a vertical metallicity gradient in the Galactic disk. The existence of a vertical gradient for field stars have been claimed by several authors, although they cover only about $1 \mathrm{kpc}$ above and below the disc plane (Bartašiūtè et al. 2003; Marsakov \& Borkova 2005, 2006; Soubiran et al. 2008). Studies covering large ranges of $|z|$ do not find any evidence of a vertical gradient (Gilmore et al. 1995; Soubiran \& Girard 2005; Navarro et al. 2011) among the field populations. Studies using OCs have found a vertical gradient of $\sim-0.3 \mathrm{dex} \mathrm{kpc}^{-1}$ (Piatti et al. 1995; Carraro et al. 1998; Chen et al. 2003), although, these studies do not distinguish the effects of the radial gradient, which can mask any vertical trend. This effects were taken into account by Jacobson et al. (2011a) who found no evidence of a vertical gradient.

To investigate the presence of trends with $z$ in our compilation, we firstly had to remove the contribution of the radial metallicity gradient. We plotted in Fig. 10 the variation in $[\mathrm{Fe} / \mathrm{H}]$ with $|z|$ in four different annuli of $R_{\mathrm{gc}}$. We note that OC with high $|z|$ are preferentially located at large $R_{\mathrm{gc}}$; this is not unexpected because the disc thickens in its external regions. Moreover, an intrinsic bias caused by obscuration in the plane appears: clusters at large Galactocentric radii are found and observed preferentially higher above the plane. This could explain why the two

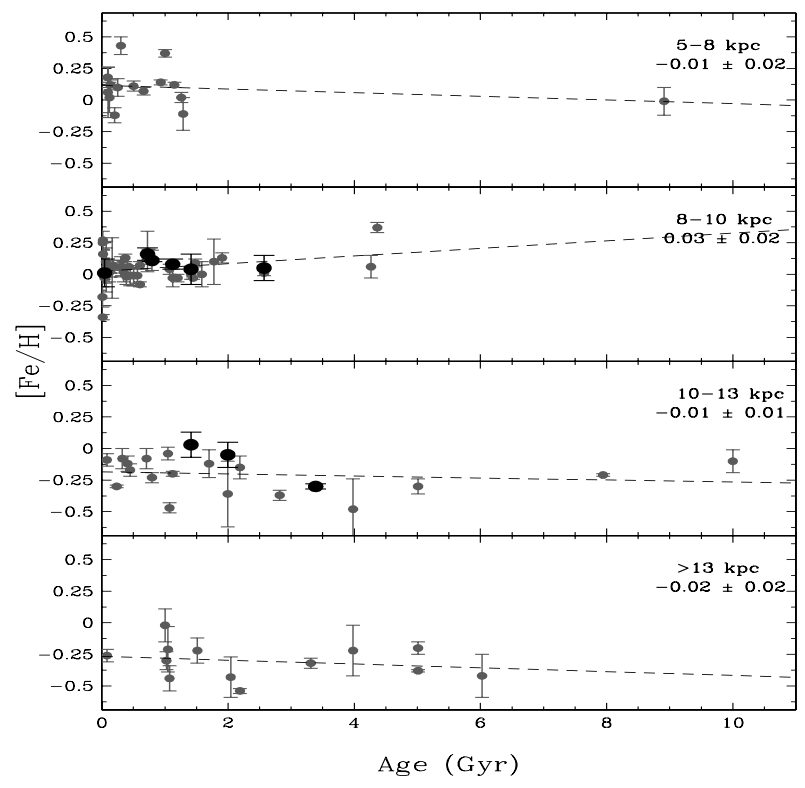

Fig. 11. The evolution of $[\mathrm{Fe} / \mathrm{H}]$ with age in the same four radial annuli as in Fig. 10. Again, dashed lines representing the median metallicity of clusters in each radial annulus have been plotted as reference.

outermost annuli studied uncover a possible weak decrease in $[\mathrm{Fe} / \mathrm{H}]$ as $z$ increases. This trend is however still compatible with no gradient at the $1 \sigma$ level and, once again, larger samples of homogeneous data are necessary to investigate this result in detail.

\subsection{Is there an age-metallicity relation for open clusters?}

Another important prediction of the chemical evolution models is the existence of an age-metallicity relation for disc populations. It is still unclear whether or not the field disc stars follow an age-metallicity relation. Some works find it (e.g., Reddy et al. 2003; Bensby et al. 2004; Reid et al. 2007), but others do not (e.g., Feltzing et al. 2001; Nordström et al. 2004; Karataş et al. 2005). Again, no clear trend of chemical abundances with age has been clearly observed in the case of Galactic OC (e.g., Friel 1995). Although Friel et al. (2010) notices a trend of $[\mathrm{Al} / \mathrm{Fe}]$ and $[\mathrm{O} / \mathrm{Fe}]$ ratios with age, again larger and homogeneous samples are necessary to confirm this result. If an age-metallicity relation is confirmed for the field population but not for OC, this would imply that they might have followed a different chemical evolution (Yong et al. 2005).

The evolution of the radial gradient as a function of time, described above, indicates that the chemical enrichment of OC is modulated by their location in the Galaxy and not by the moment at which they formed. To investigate whether an age-metallicity relationship exits at a given $R_{\mathrm{gc}}$, we plotted in Fig. 11 the evolution of $[\mathrm{Fe} / \mathrm{H}]$ with age in four different radial annuli. There is no clear trend in any of the studied annuli, although not all of them contain clusters covering the same age range. Only in the outermost annulus is a weak trend observed, although it is still not very significant. Again, we conclude that a larger sample of homogeneous data are necessary to investigate this point in depth.

\section{Summary and conclusions}

We have enlarged our sample of homogeneous high-resolution abundance measurements from the five clusters of Paper I to a 
total of nine, analysing here spectra of red clump giants in the Hyades, Praesepe, NGC 752, and Be 32. Our main results can be summarized as follows:

- We provide the first high-resolution based abundance ratios (other than $[\mathrm{Fe} / \mathrm{H}]$, see Sestito et al. 2008) for NGC 752, which turned out to be mostly of solar composition.

- We have presented the abundance ratios of Praesepe red clump giants, which appear to solve a puzzling dichotomy of literature determinations for stars of different evolutionary stages.

- We have found that our abundance ratios for the Hyades and Berkeley 32 are in good agreement with other literature determinations.

- We have confirmed the absence of light elements (anti-)correlations in the OC studied so far.

We have updated our compilation of previous literature data for 57 clusters of Paper I to a total of 89 clusters presented here. With this updated compilation and our homogeneous measurements in hand, we have investigated Galactic trends in $[\mathrm{Fe} / \mathrm{H}]$ (and $[\alpha / \mathrm{Fe}]$ ) with age, Galactocentric radius, and height above the Galactic plane. Our findings are in substantial agreement with other similar investigations, where the abundance gradient appears to indeed flatten out outside $R_{\mathrm{gc}} \simeq 12.5 \mathrm{kpc}$, and the inner disc slope appears to flatten for younger ages as well, although the age bins are not too well-sampled. At the same time, $[\alpha / \mathrm{Fe}]$ shows a weak increase with $R_{\mathrm{gc}}$. No significant gradients are observed with $|z|$ or age, except for a weak tendency of $[\mathrm{Fe} / \mathrm{H}]$ to decrease with increasing $|z|$ and decrease with age in the outermost disc annulus studied. None of our measured weak trends have any significance above $1 \sigma$. Larger samples of homogeneous data are still necessary to investigate the existence of any dependence on age and $|z|$ in the Galactic disc.

Acknowledgements. We acknowledge the anonymous referee for helping us to improve this paper. R.C. acknowledge the support from the Spanish Ministry of Science and Technology (Plan Nacional de Investigación Científica, Desarrollo, e Investigación Tecnológica, AYA2004-06343 and AYA2007-3E3507). R.C. also acknowledges the funds by the Spanish Ministry of Science and Innovation under the Juan de la Cierva and MEC/Fullbrigth fellowships, and by the Centro de Investigaciones de Astronomía (Venezuela) under its postdoctoral fellowship programe.

\section{References}

Alonso, A., Arribas, S., \& Martínez-Roger, C. 1999, A\&AS, 140, 261 An, D., Terndrup, D. M., Pinsonneault, M. H., et al. 2007, ApJ, 655, 233 Andersen, J. 1999, Transactions of the IAU, 24, A36

Andreuzzi, G., Bragaglia, A., Tosi, M., \& Marconi, G. 2011, MNRAS, 412, 1265 Andrievsky, S. M., Luck, R. E., Martin, P., \& Lépine, J. R. D. 2004, A\&A, 413, 159

Arp, H. 1962, ApJ, 136, 66

Barry, D. C., Cromwell, R. H., \& Hege, E. K. 1987, ApJ, 315, 264

Bartašiūtè, S., Aslan, Z., Boyle, R. P., et al. 2003, Balt. Astron., 12, 539

Bartašiūtè, S., Deveikis, V., Straižys, V., \& Bogdanovičius, A. 2007, Balt. Astron., 16, 199

Bensby, T., Feltzing, S., \& Lundström, I. 2004, A\&A, 421, 969

Bessell, M. S. 1979, PASP, 91, 589

Blake, R. M. 2002, Ph.D. Thesis,

Blake, R. M., \& Rucinski, S. M. 2004, BAAS, 36, 1483

Boesgaard, A. M. 1989, ApJ, 336, 798

Boesgaard, A. M. 1991, ApJ, 370, L95

Boesgaard, A. M., \& Friel, E. D. 1990, ApJ, 351, 467

Boesgaard, A. M., Jensen, E. E. C., \& Deliyannis, C. P. 2009, AJ, 137, 4949

Bouvier, J., Kendall, T., Meeus, G., et al. 2008, A\&A, 481, 661
Boyarchuk, A. A., Antipova, L. I., Boyarchuk, M. E., \& Savanov, I. S. 2000, Astron. Rep., 44, 76

Bragaglia, A., Carretta, E., Gratton, R. G., et al. 2001, AJ, 121, 327

Bragaglia, A., Sestito, P., Villanova, S., et al. 2008, A\&A, 480, 79

Brown, J. A., Wallerstein, G., Geisler, D., \& Oke, J. B. 1996, AJ, 112, 1551

Burkhart, C., \& Coupry, M. F. 1998, A\&A, 338, 1073

Burkhart, C., \& Coupry, M. F. 2000, A\&A, 354, 216

Cardelli, J. A., Clayton, G. C., \& Mathis, J. S. 1989, ApJ, 345, 245

Carraro, G., \& Bensby, T. 2009, MNRAS, 397, L106

Carraro, G., \& Chiosi, C. 1994, A\&A, 287, 761

Carraro, G., Bertelli, G., Bressan, A., \& Chiosi, C. 1993, A\&AS, 101, 381

Carraro, G., Ng, Y. K., \& Portinari, L. 1998, MNRAS, 296, 1045

Carraro, G., Bresolin, F., Villanova, S., et al. 2004, AJ, 128, 1676

Carraro, G., Villanova, S., Demarque, P., et al. 2006, ApJ, 643, 1151

Carraro, G., de La Fuente Marcos, R., Villanova, S., et al. 2007a, A\&A, 466, 931

Carraro, G., Geisler, D., Villanova, S., Frinchaboy, P. M., \& Majewski, S. R. 2007b, A\&A, 476, 217

Carraro, G., Villanova, S., Demarque, P., Moni Bidin, C., \& McSwain, M. V. 2008, MNRAS, 386, 1625

Carretta, E., Bragaglia, A., Gratton, R. G., \& Tosi, M. 2004, A\&A, 422, 951

Carretta, E., Bragaglia, A., Gratton, R. G., \& Tosi, M. 2005, A\&A, 441, 131

Carretta, E., Bragaglia, A., \& Gratton, R. G. 2007, A\&A, 473, 129

Carretta, E., Bragaglia, A., Gratton, R. G., et al. 2010, A\&A, 516, A55

Cayrel, R., Depagne, E., Spite, M., et al. 2004, A\&A, 416, 1117

Chen, L., Hou, J. L., \& Wang, J. J. 2003, AJ, 125, 1397

Chiappini, C., Matteucci, F., \& Romano, D. 2001, ApJ, 554, 1044

Claria, J. J., Piatti, A. E., \& Osborn, W. 1996, PASP, 108, 672

Coleman, L. A. 1982, AJ, 87, 369

Costa, R. D. D., Uchida, M. M. M., \& Maciel, W. J. 2004, A\&A, 423, 199

Crawford, D. L., \& Barnes, J. V. 1970, AJ, 75, 946

Cutri, R. M., Skrutskie, M. F., Van Dyk, et al. 2003, Explanatory Supplement to the 2MASS All Sky Data Release, http://www.ipac.caltech.edu/ 2mass/releases/allsky/doc/explsup.html

Daflon, S., \& Cunha, K. 2004, ApJ, 617, 1115

Daniel, S. A., Latham, D. W., Mathieu, R. D., \& Twarog, B. A. 1994, PASP, 106, 281

Dean, J. F., Warren, P. R., \& Cousins, A. W. J. 1978, MNRAS, 183, 569

Deharveng, L., Peña, M., Caplan, J., \& Costero, R. 2000, MNRAS, 311, 329

Den Hartog, E. A., Lawler, J. E., Sneden, C., \& Cowan, J. J. 2003, ApJS, 148, 543

De Silva, G. M., Sneden, C., Paulson, D. B., et al. 2006, AJ, 131, 455

De Silva, G. M., Freeman, K. C., Asplund, M., et al. 2007, AJ, 133, 1161

de Silva, G. M., Gibson, B. K., Lattanzio, J., \& Asplund, M. 2009, A\&A, 500, L25

Dias, W. S., Alessi, B. S., Moitinho, A., \& Lépine, J. R. D. 2002, A\&A, 389, 871

Dinescu, D. I., Demarque, P., Guenther, D. B., \& Pinsonneault, M. H. 1995, AJ, 109,2090

D’Orazi, V., \& Randich, S. 2009, A\&A, 501, 553

D’Orazi, V., Bragaglia, A., Tosi, M., Di Fabrizio, L., \& Held, E. V. 2006, MNRAS, 368, 471

D’Orazi, V., Magrini, L., Randich, S., et al. 2009, ApJ, 693, L31

Dutra, C. M., \& Bica, E. 2000, A\&A, 359, 347

Dzervitis, U., \& Paupers, O. 1993, Ap\&SS, 199, 77

Edvardsson, B., Andersen, J., Gustafsson, B., et al. 1993, A\&A, 275, 101

Edvardsson, B., Pettersson, B., Kharrazi, M., \& Westerlund, B. 1995, A\&A, 293, 75

Eggen, O. J. 1963, ApJ, 138, 356

Eggen, O. J. 1989, PASP, 101, 54

Eggen, O. J. 1998, AJ, 116, 284

Famaey, B., Jorissen, A., Luri, X., et al. 2005, A\&A, 430, 165

Famaey, B., Pont, F., Luri, X., et al. 2007, A\&A, 461, 957

Feltzing, S., Holmberg, J., \& Hurley, J. R. 2001, A\&A, 377, 911

Ford, A., Jeffries, R. D., \& Smalley, B. 2005, MNRAS, 364, 272

Fossati, L., Folsom, C. P., Bagnulo, S., et al. 2011, MNRAS, 413, 1132

Francic, S. P. 1989, AJ, 98, 888

Friel, E. D. 1995, ARA\&A, 33, 381

Friel, E. D., \& Boesgaard, A. M. 1992, ApJ, 387, 170

Friel, E. D., \& Janes, K. A. 1993, A\&A, 267, 75

Friel, E. D., Janes, K. A., Tavarez, M., et al. 2002, AJ, 124, 2693

Friel, E. D., Jacobson, H. R., Barrett, E., et al. 2003, AJ, 126, 2372

Friel, E. D., Jacobson, H. R., \& Pilachowski, C. A. 2005, AJ, 129, 2725

Friel, E. D., Jacobson, H. R., \& Pilachowski, C. A. 2010, AJ, 139, 1942

Frinchaboy, P. M., Marino, A. F., Villanova, S., et al. 2008, MNRAS, 391, 39

Fulbright, J. P., McWilliam, A., \& Rich, R. M. 2007, ApJ, 661, 1152

Gáspár, A., Rieke, G. H., Su, K. Y. L., et al. 2009, ApJ, 697, 1578

Gebran, M., \& Monier, R. 2008, A\&A, 483, 567

Gebran, M., Monier, R., \& Richard, O. 2008, A\&A, 479, 189

Giardino, G., Pillitteri, I., Favata, F., \& Micela, G. 2008, A\&A, 490, 113 
Gilmore, G., Wyse, R. F. G., \& Jones, J. B. 1995, AJ, 109, 1095

Girardi, L., \& Salaris, M. 2001, MNRAS, 323, 109

Girardi, L., Mermilliod, J.-C., \& Carraro, G. 2000, A\&A, 354, 892

Gonzalez, G., \& Lambert, D. L. 1996, AJ, 111, 424

Gonzalez, G., \& Wallerstein, G. 2000, PASP, 112, 1081

Gratton, R. G., \& Contarini, G. 1994, A\&A, 283, 911

Gratton, R. G., Carretta, E., Eriksson, K., \& Gustafsson, B. 1999, A\&A, 350, 955

Grevesse, N., Noels, A., \& Sauval, A. J. 1996, Cosmic Abundances, ASP Conf. Ser., 99,117

Griffin, R. F., Griffin, R. E. M., Gunn, J. E., \& Zimmerman, B. A. 1988, AJ, 96, 172

Hamdani, S., North, P., Mowlavi, N., Raboud, D., \& Mermilliod, J.-C. 2000, A\&A, 360, 509

Hardy, E. 1979, AJ, 84, 319

Heinemann, K. 1926, Astron. Nachr., 227, 193

Hernandez, M. M., Perez Hernandez, F., Michel, E., et al. 1998, A\&A, 338, 511 Hertzsprung, E. 1909, ApJ, 30, 135

Hill, V., \& Pasquini, L. 1999, A\&A, 348, L21

Hobbs, L. M., \& Thorburn, J. A. 1992, AJ, 104, 669

Høg, E., Fabricius, C., Makarov, V. V., et al. 2000, A\&A, 355, L27

Hui-Bon-Hoa, A., \& Alecian, G. 1998, A\&A, 332, 224

Jacobson, H. R., Friel, E. D., \& Pilachowski, C. A. 2007, AJ, 134, 1216

Jacobson, H. R., Friel, E. D., \& Pilachowski, C. A. 2008, AJ, 135, 2341

Jacobson, H. R., Friel, E. D., \& Pilachowski, C. Abartasiute200. 2009, AJ, 137, 4753

Jacobson, H. R., Friel, E. D., \& Pilachowski, C. A. 2011a, AJ, 141, 58

Jacobson, H. R., Pilachowski, C. A., \& Friel, E. D. 2011b, AJ, 142, 59

Jacquinet-Husson, N., Ari, E., Ballard, J., et al. 1999, JQSRT, 62, 205

Jacquinet-Husson, N., Scott, N. A., Garceran, K., Armante, R., \& Chédin, A. 2005, JQSRT, 95, 429

Jameson, R. F., Lodieu, N., Casewell, S. L., Bannister, N. P., \& Dobbie, P. D. 2008, MNRAS, 385, 1771

Janes, K. A. 1979, ApJS, 39, 135

Jennens, P. A., \& Helfer, H. L. 1975, MNRAS, 172, 681

Johansson, S., Litzén, U., Lundberg, H., \& Zhang, Z. 2003, ApJ, 584, L107

Johnson, H. L. 1952, ApJ, 116, 640

Johnson, H. L. 1953, ApJ, 117, 356

Johnson, H. L. 1961, Lowell Observatory Bulletin, 5, 133

Johnson, H. L., \& Knuckles, C. F. 1955, ApJ, 122, 209

Johnson, H. L., Iriarte, B., Mitchell, R. I., \& Wisniewskj, W. Z. 1966,

Communications of the Lunar and Planetary Laboratory, 4, 99

Kaluzny, J., \& Mazur, B. 1991, Acta Astron., 41, 167

Karataș, Y., Bilir, S., \& Schuster, W. J. 2005, MNRAS, 360, 1345

King, J. R., Soderblom, D. R., Fischer, D., \& Jones, B. F. 2000, ApJ, 533, 944

Klein Wassink, W. J. 1927, Publications of the Kapteyn Astronomical Laboratory Groningen, 41, 1

Komarov, N. S., \& Basak, N. Y. 1993, AZh, 70, 111

Kraus, A. L., \& Hillenbrand, L. A. 2007, AJ, 134, 2340

Kupka, F., Piskunov, N., Ryabchikova, T. A., Stempels, H. C., \& Weiss, W. W. 1999, A\&AS, 138, 119

Lata, S., Pandey, A. K., Sagar, R., \& Mohan, V. 2002, A\&A, 388, 158

Lemasle, B., François, P., Piersimoni, A., et al. 2008, A\&A, 490, 613

Letarte, B., Hill, V., Jablonka, P., et al. 2006, A\&A, 453, 547

Loktin, A. V. 2000, Astron. Lett., 26, 657

Loktin, A. V., \& Beshenov, G. V. 2001, Astron. Lett., 27, 386

Luck, R. E. 1994, ApJS, 91, 309

Luck, R. E., \& Challener, S. L. 1995, AJ, 110, 2968

Maciel, W. J., \& Costa, R. D. D. 2009, IAU Symp., 254, 38P

Maciel, W. J., Costa, R. D. D., \& Uchida, M. M. M. 2003, A\&A, 397, 667

Maciel, W. J., Quireza, C., \& Costa, R. D. D. 2007, A\&A, 463, L13

Maeder, A. 1971, A\&A, 10, 354

Magain, P. 1984, A\&A, 134, 189

Magrini, L., Sestito, P., Randich, S., \& Galli, D. 2009, A\&A, 494, 95

Magrini, L., Randich, S., Zoccali, M., et al. 2010, A\&A, 523, 11

Mallik, S. V. 1998, A\&A, 338, 623

Marsakov, V. A., \& Borkova, T. V. 2005, Astron. Lett., 31, 515

Marsakov, V. A., \& Borkova, T. V. 2006, Astron. Lett., 32, 376

Martell, S. L., \& Grebel, E. K. 2010, A\&A, 519, A14

Martell, S. L., \& Smith, G. H. 2009, PASP, 121, 577

Mathieu, R. D., \& Mazeh, T. 1988, ApJ, 326, 256

Mazzei, P., \& Pigatto, L. 1988, A\&A, 193, 148

Mendoza, E. E. 1967, Boletín de los Observatorios Tonantzintla y Tacubaya, 4, 149

Mermilliod, J.-C. 1995, Information \& On-Line Data in Astronomy, ASSL, 203, 127

Mermilliod, J.-C., Mathieu, R. D., Latham, D. W., \& Mayor, M. 1998, A\&A, 339,423
Mermilliod, J.-C., Andersen, J., Latham, D. W., \& Mayor, M. 2007, A\&A, 473, 829

Meynet, G., Mermilliod, J.-C., \& Maeder, A. 1993, A\&AS, 98, 477

Mikolaitis, Š., Tautvaišienè, G., Gratton, R., Bragaglia, A., \& Carretta, E. 2010, MNRAS, 407, 1866

Mikolaitis, Š., Tautvaišienė, G., Gratton, R., Bragaglia, A., \& Carretta, E. 2011a, MNRAS, 413, 2199

Mikolaitis, Š., Tautvaišienė, G., Gratton, R., Bragaglia, A., \& Carretta, E. 2011b, MNRAS, 416, 1092

Milone, E. F., Stagg, C. R., Sugars, B. A., et al. 1995, AJ, 109, 359

Mishenina, T. V., Bienaymé, O., Gorbaneva, T. I., et al. 2006, A\&A, 456, 1109

Mishenina, T. V., Gorbaneva, T. I., Bienaymé, O., et al. 2007, Astron. Rep., 51, 382

Molla, M., Ferrini, F., \& Diaz, A. I. 1996, ApJ, 466, 668

Montegriffo, P., Ferraro, F. R., Origlia, L., \& Fusi Pecci, F. 1998, MNRAS, 297, 872

Monroe, T. R., \& Pilachowski, C. A. 2010, AJ, 140, 2109

Mould, J. 2005, AJ, 129, 698

Mucciarelli, A. 2011, A\&A, 528, 44

Mucciarelli, A., Origlia, L., Ferraro, F. R., \& Pancino, E. 2009, ApJ, 695, L134

Narayanan, V. K., \& Gould, A. 1999, ApJ, 515, 256

Navarro, J. F., Abadi, M. G., Venn, K. A., Freeman, K. C., \& Anguiano, B. 2011, MNRAS, 412, 1203

Nicolet, B. 1981, A\&A, 104, 185

Nissen, P. E. 1988, A\&A, 199, 146

Nordström, B., Mayor, M., Andersen, J., et al. 2004, A\&A, 418, 989

Origlia, L., Valenti, E., Rich, R. M., \& Ferraro, F. R. 2006, ApJ, 646, 499

Pace, G., Pasquini, L., \& François, P. 2008, A\&A, 489, 403

Pace, G., Danziger, J., Carraro, G., et al. 2010, A\&A, 515, A28

Pancino, E., Carrera, R., Rossetti, E., \& Gallart, C. 2010a, A\&A, 511, A56 (Paper I)

Pancino, E., Rejkuba, M., Zoccali, M., \& Carrera, R. 2010b, A\&A, 524, A44

Pasquini, L., Randich, S., Zoccali, M., et al. 2004, A\&A, 424, 951

Patenaude, M. 1978, A\&A, 66, 225

Paulson, D. B., Sneden, C., \& Cochran, W. D. 2003, AJ, 125, 3185

Pereira, C. B., \& Quireza, C. 2010, IAU Symp., 266, 495

Percival, S. M., Salaris, M., \& Kilkenny, D. 2003, A\&A, 400, 541

Perryman, M. A. C., Lindegren, L., Kovalevsky, J., et al. 1997, A\&A, 323, L49

Perryman, M. A. C., Brown, A. G. A., Lebreton, Y., et al. 1998, A\&A, 331, 81

Peterson, R. C., \& Green, E. M. 1998, ApJ, 502, L39

Pfeiffer, M. J., Frank, C., Baumueller, D., Fuhrmann, K., \& Gehren, T. 1998, A\&AS, 130, 381

Piatti, A. E., Claria, J. J., \& Abadi, M. G. 1995, AJ, 110, 2813

Pinsonneault, M. H., Stauffer, J., Soderblom, D. R., King, J. R., \& Hanson, R. B. 1998, ApJ, 504, 170

Platais, I., Melo, C., Fulbright, J. P., et al. 2008, MNRAS, 391, 1482

Pourbaix, D., Tokovinin, A. A., Batten, A. H., et al. 2004, A\&A, 424, 727

Ramírez, S. V., \& Cohen, J. G. 2003, AJ, 125, 224

Randich, S., Pallavicini, R., Meola, G., Stauffer, J. R., \& Balachandran, S. C. 2001, A\&A, 372, 862

Randich, S., Sestito, P., \& Pallavicini, R. 2003, A\&A, 399, 133

Randich, S., Sestito, P., Primas, F., Pallavicini, R., \& Pasquini, L. 2006, A\&A, 450,557

Reddy, B. E., Tomkin, J., Lambert, D. L., \& Allende Prieto, C. 2003, MNRAS, 340,304

Reddy, B. E., Lambert, D. L., \& Allende Prieto, C. 2006, MNRAS, 367, 1329

Reid, I. N., Turner, E. L., Turnbull, M. C., Mountain, M., \& Valenti, J. A. 2007, ApJ, 665, 767

Richtler, T., \& Sagar, R. 2001, Bull. Astron. Soc. India, 29, 53

Roman, N. G. 1955, ApJ, 121, 454

Rohlfs, K., \& Vanysek, V. 1962, Astronomische Abhandlungen der Hamburger Sternwarte, 5, 341

Roškar, R., Debattista, V. P., Quinn, T. R., Stinson, G. S., \& Wadsley, J. 2008, ApJ, 684, L79

Salaris, M., Weiss, A., \& Percival, S. M. 2004, A\&A, 414, 163

Santos, N. C., Lovis, C., Pace, G., Melendez, J., \& Naef, D. 2009, A\&A, 493, 309

Schuler, S. C., King, J. R., Fischer, D. A., Soderblom, D. R., \& Jones, B. F. 2003, AJ, 125,2085

Schuler, S. C., Hatzes, A. P., King, J. R., Kürster, M., \& The, L.-S. 2006, AJ, 131,1057

Schuler, S. C., King, J. R. \& The, L.-S. 2009, ApJ, 701, 837

Sestito, P., Randich, S., Mermilliod, J.-C., \& Pallavicini, R. 2003, A\&A, 407, 289

Sestito, P., Randich, S., \& Pallavicini, R. 2004, A\&A, 426, 809

Sestito, P., Bragaglia, A., Randich, S., et al. 2006, A\&A, 458, 121

Sestito, P., Randich, S., \& Bragaglia, A. 2007, A\&A, 465, 185 
R. Carrera and E. Pancino: Abundances of four open clusters

Sestito, P., Bragaglia, A., Randich, S., et al. 2008, A\&A, 488, 943

Schaye, J. 2004, ApJ, 609, 667

Shen, Z.-X., Jones, B., Lin, D. N. C., Liu, X.-W., \& Li, S.-L. 2005, ApJ, 635, 608

Skrutskie, M. F., Cutri, R. M., Stiening, R., et al. 2006, AJ, 131, 1163

Smiljanic, R., Gauderon, R., North, P., et al. 2009, A\&A, 502, 267

Smith, V. V., \& Suntzeff, N. B. 1987, AJ, 93, 359

Soderblom, D. R., Laskar, T., Valenti, J. A., Stauffer, J. R., \& Rebull, L. M. 2009, AJ, 138, 1292

Soubiran, C., \& Girard, P. 2005, A\&A, 438, 139

Soubiran, C., Bienaymé, O., Mishenina, T. V., \& Kovtyukh, V. V. 2008, A\&A, 480, 91

Spite, M. 1967, Annales d'Astrophysique, 30, 211

Stanghellini, L., \& Haywood, M. 2010, ApJ, 714, 1096

Stetson, P. B., \& Pancino, E. 2008, PASP, 120, 1332

Tadross, A. L. 2001, New Astron., 6, 293

Tautvaiŝiiene, G., Edvardsson, B., Tuominen, I., \& Ilyin, I. 2000, A\&A, 360, 499

Tautvaišienè, G., Edvardsson, B., Puzeras, E., \& Ilyin, I. 2005, A\&A, 431, 933
Taylor, B. J. 2006, AJ, 132, 2453

Taylor, B. J. 2007, AJ, 134, 934

Terndrup, D. M., Pinsonneault, M., Jeffries, R. D., et al. 2002, ApJ, 576, 950

Tosi, M., Bragaglia, A., \& Cignoni, M. 2007, MNRAS, 378, 730

Tsvetkov, T. G. 1993, Ap\&SS, 203, 247

Twarog, B. A. 1983, ApJ, 267, 207

Twarog, B. A., Ashman, K. M., \& Anthony-Twarog, B. J. 1997, AJ, 114, 2556

Twarog, B. A., Anthony-Twarog, B. J., \& De Lee, N. 2003, AJ, 125, 1383

van Bueren, H. G. 1952, Bull. Astron. Inst. Netherlands, 11, 385

van den Heuvel, E. P. J. 1969, PASP, 81, 815

van Leeuwen, F. 1999, A\&A, 341, L71

Varenne, O., \& Monier, R. 1999, A\&A, 351, 247

Villanova, S., Carraro, G., Bresolin, F., \& Patat, F. 2005, AJ, 130, 652

Villanova, S., Baume, G., \& Carraro, G. 2007, MNRAS, 379, 1089

Villanova, S., Carraro, G., \& Saviane, I. 2009, A\&A, 504, 845

Villanova, S., Geisler, D., \& Piotto, G. 2010, ApJ, 722, L18

Yong, D., Carney, B. W., \& Texeira de Almeida, M. L. 2005, AJ, 130, 597 


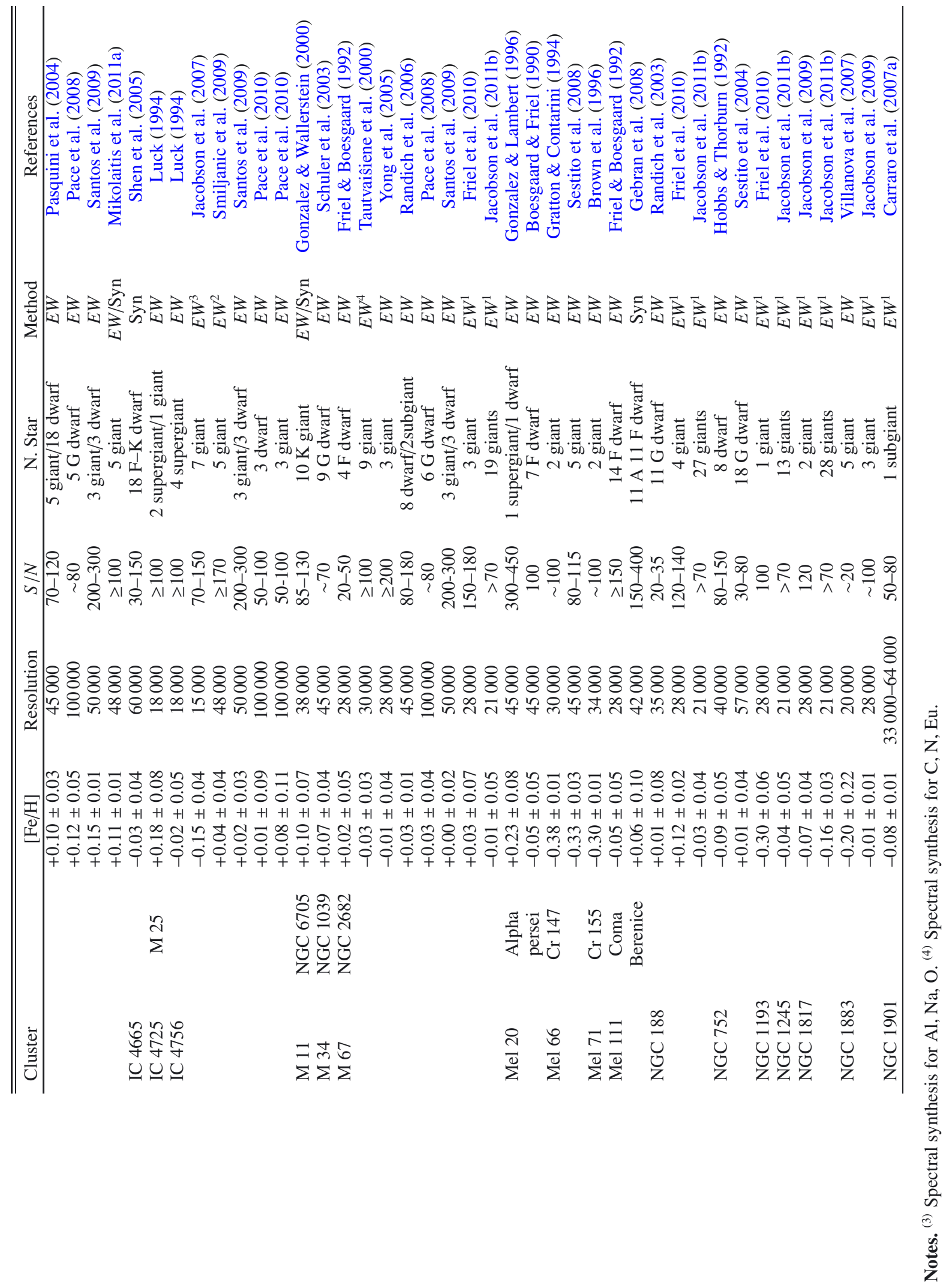




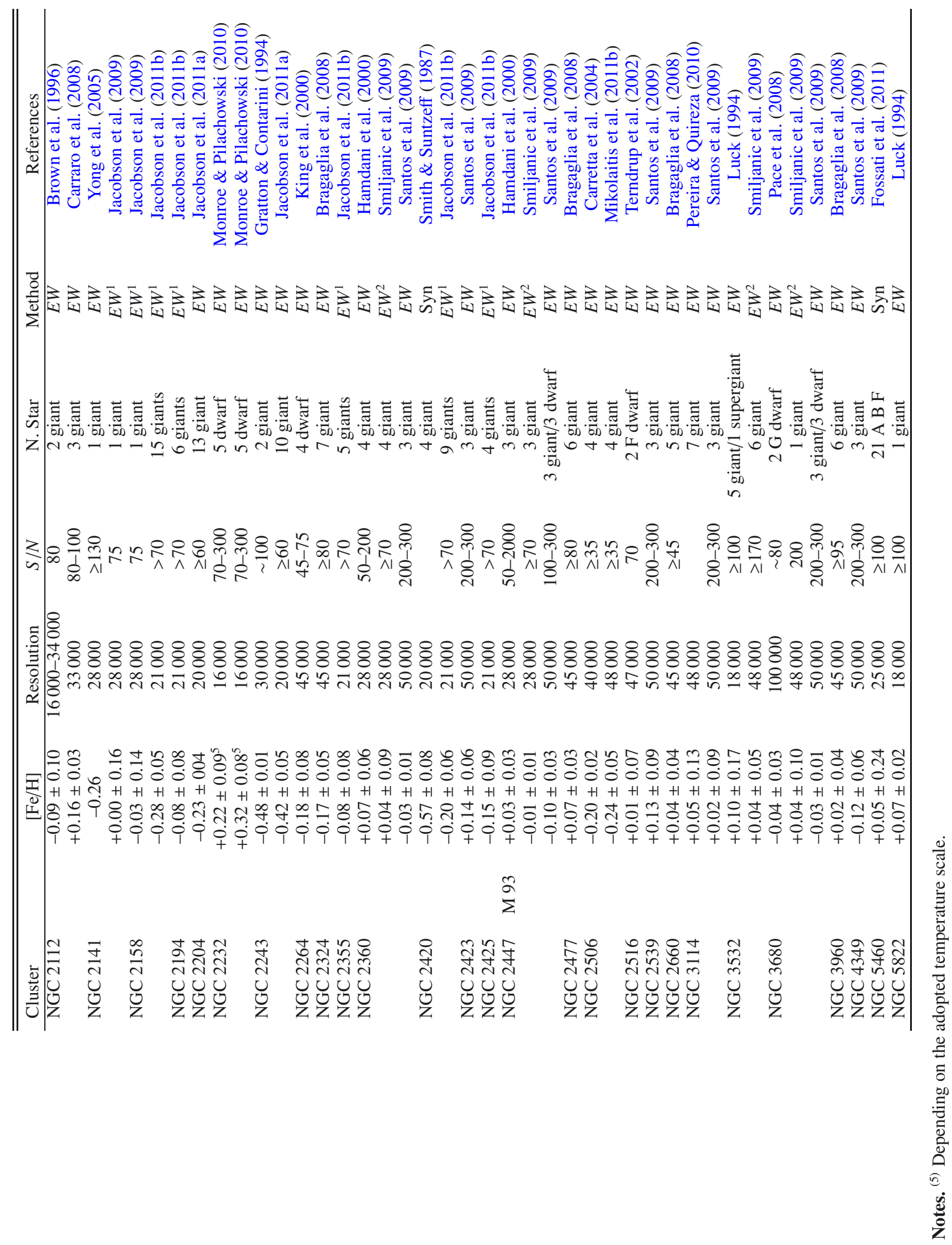




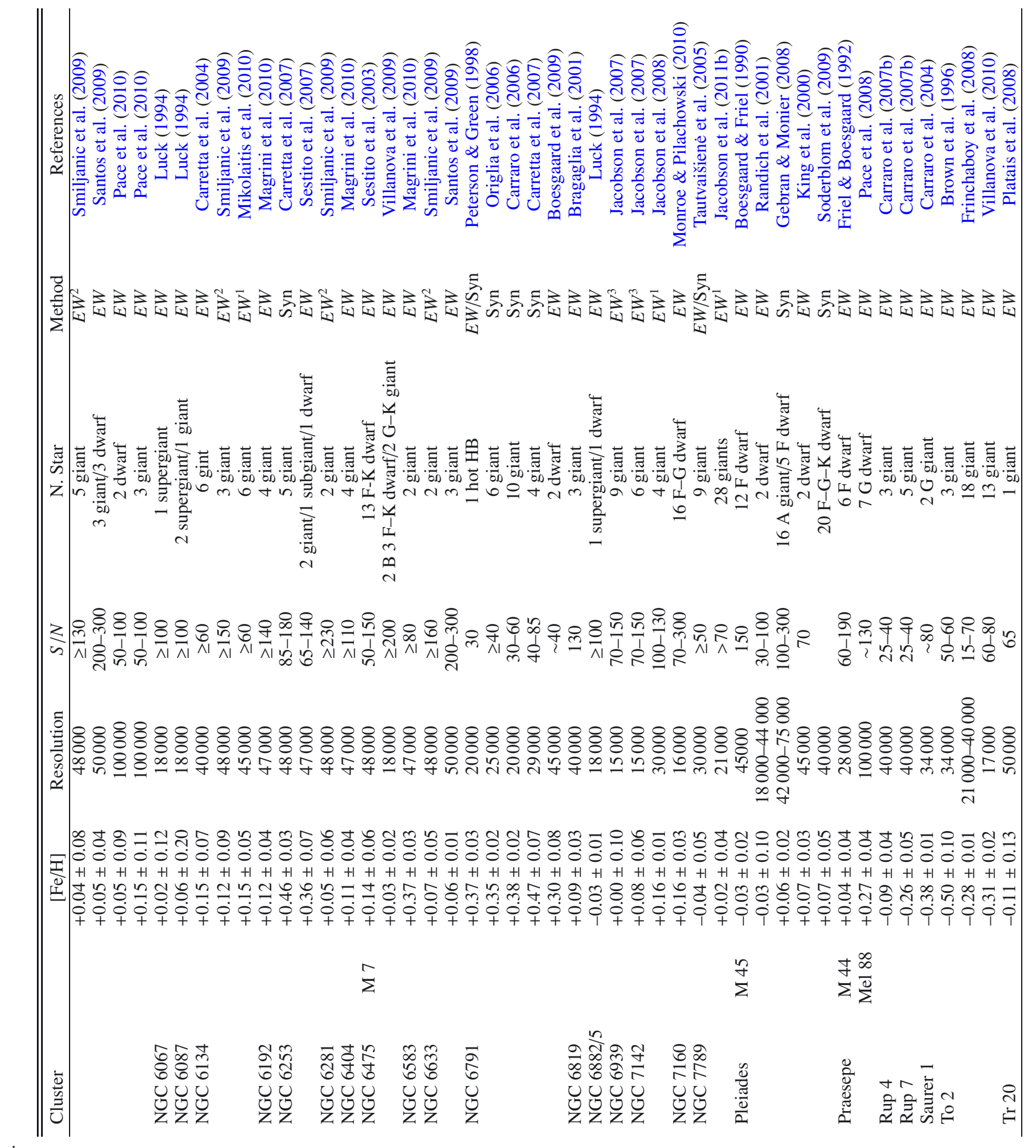

